\title{
Multiscale Friction Simulation of Dry Polymer Contacts: Reaching Experimental Length Scales by Coupling Molecular Dynamics and Contact Mechanics
}

\section{Daniele Savio ( $\sim$ Daniele.Savio@freudenberg.com )}

Freudenberg Technology Innovation SE \&amp; Co. KG https://orcid.org/0000-0003-1908-2379 Jannik Hamann

Fraunhofer Institute for Mechanics of Materials: Fraunhofer-Institut fur Werkstoffmechanik IWM

\section{Pedro A. Romero}

Freudenberg Technology Innovation SE \&amp; Co. KG

\section{Christoph Klingshirn}

Freudenberg FST GmbH

Ravindrakumar Bactavatchalou

Freudenberg Technology Innovation SE \&amp; Co. KG

\section{Martin Dienwiebel}

Fraunhofer Institute for Mechanics of Materials: Fraunhofer-Institut fur Werkstoffmechanik IWM

\section{Michael Moseler}

Fraunhofer Institute for Mechanics of Materials: Fraunhofer-Institut fur Werkstoffmechanik IWM

\section{Research Article}

Keywords: Coupling Molecular Dynamics, Contact Mechanics, Multiscale Friction

Posted Date: February 23rd, 2021

DOI: https://doi.org/10.21203/rs.3.rs-223573/v1

License: (9) (i) This work is licensed under a Creative Commons Attribution 4.0 International License. Read Full License 


\title{
Multiscale friction simulation of dry polymer contacts: reaching experimental length scales by coupling molecular dynamics and contact mechanics
}

\author{
Daniele Savio $^{1 *}$, Jannik Hamann ${ }^{2}$, Pedro A. Romero ${ }^{1}$, Christoph Klingshirn ${ }^{3}$, Ravindrakumar \\ Bactavatchalou $^{1}$, Martin Dienwiebel ${ }^{2,4}$, Michael Moseler ${ }^{2,5}$ \\ ${ }^{1}$ Freudenberg Technology Innovation SE \& Co. KG, Weinheim, Germany \\ ${ }^{2} \mu$ TC Microtribology Center, Fraunhofer Institute for Mechanics of Materials IWM, Freiburg, Germany \\ ${ }^{3}$ Freudenberg FST GmbH, Weinheim, Germany \\ ${ }^{4}$ Karlsruhe Institute of Technology, Institute for Applied Materials, IAM-CMS, Karlsruhe, Germany \\ ${ }^{5}$ Institute of Physics, University of Freiburg, Freiburg, Germany \\ *Corresponding author: daniele.savio@freudenberg.com
}

\begin{abstract}
This work elucidates friction in Poly-Ether-Ether-Ketone (PEEK) sliding contacts through multiscale simulations. At the nanoscale, non-reactive classical molecular dynamics (MD) simulations of dry and water-lubricated amorphous PEEK-PEEK interfaces are performed. During a short running-in phase, we observe structural transformations at the sliding interface that result in flattening of the initial nanotopographies accompanied by strong polymer chain alignment in the shearing direction. Our MD simulations reveal a linear pressure-dependence of the shear stress $\bar{\tau}_{M D}\left(P, \sigma_{\mathrm{H}_{2} \mathrm{O}}\right)[M P a]=0.18 \mathrm{P}+50.5-1.25 \sigma_{\mathrm{H}_{2} \mathrm{O}}$, where $\sigma_{\mathrm{H}_{2} \mathrm{O}}\left[\mathrm{nm}^{-2}\right]$ is the surface number density of adsorbed water molecules. This constitutive law is of central importance for our multiscale approach, since it forms a link between MD and elastoplastic contact mechanics calculations. An integration of $\bar{\tau}_{M D}\left(P, \sigma_{\mathrm{H}_{2} \mathrm{O}}\right)$ over the real area of contact yields a macroscopic friction coefficient $\mu_{\text {macro }}\left(\sigma_{\mathrm{H}_{2} \mathrm{O}}\right)$ that allows for a meaningful comparison with friction coefficients $\mu_{\text {exp }}$ observed in macroscopic tribometer experiments. Severe normal loading conditions result in significant wear and high experimental friction coefficients $\mu_{\text {exp }} \approx 0.5-0.7$ which are in good agreement with the calculated dry friction coefficients $\mu_{\text {macro }}\left(\sigma_{\mathrm{H}_{2} \mathrm{O}}=0\right)$. For milder experimental loads, our multiscale model suggests that the lower friction states with $\mu_{\text {exp }} \approx 0.2$ originate in the presence of physisorbed molecules (e.g. water), which significantly reduce interfacial adhesion.
\end{abstract}

\section{Introduction}

Nowadays, polymers are increasingly used in tribological applications due to their advantageous properties of low friction, corrosion resistance, biocompatibility, and cost effectiveness. In this framework, a quantitative understanding of friction between polymer interfaces is fundamental to optimize their application range and improve their lifetime.

However, a numerical modelling of polymer friction remains a challenging problem, since several contributions such as adhesion, viscoelastic and thermal effects, as well as plastic processes and ploughing must be considered. Furthermore, changes in material properties e.g. strain hardening and direction-dependent transformations can occur under loading and sliding. Continuum theories and numerical calculations allow us to consider several aspects of polymer friction at the macroscopic scale [1]. Here, a key element is the real area of contact between rough surfaces [2], which can be extracted from either experiments or contact mechanics calculations $[3,4]$. In the contact spots between polymer and a countersurface, sliding is opposed by the shear strength $\tau$ of the adhesive junctions [5]. Accurate empirical estimates of this quantity require carefully chosen experimental conditions - even at model interfaces $[3,6]$. The 
underlying physical processes are also unclear, as shown by several competing models in the literature $[5,7,8]$. Furthermore, the shear strength at the contact spots strongly depends not only on the polymer and counter material but also on the presence of moisture, lubricants or transfer layers $[9,10,11]$ at the shearing interface. This makes a direct experimental determination of $\tau$ inaccessible for relevant technical surface pairings.

Atomistic simulations represent an alternative to gain insight into the tribological response of polymer interfaces. Classical molecular dynamics (MD) allows for a detailed understanding of structural transformations occurring under sliding and their relationship with friction in several classes of materials. For polymers, pioneering MD sliding simulations of PTFE and PE $[12,13,14]$ analyzed in detail the possible wear mechanisms occurring at the molecular level. MD has also been applied to relate material properties to the bulk mechanical response $[15,16]$ in order to investigate interaction of oligomer chains with fillers [17,18] and counter surfaces [19] under shear, or to simulate scratching behavior [20].

Despite the vast insights provided by MD approaches, these remain computationally expensive tools leading to a sizeable gap in length and time scales between simulations and experiments or typical applications. Efforts should therefore be made to correctly transfer the information gained from atomistic simulations into macroscopic systems, and to validate corresponding theoretical predictions by experimental observations.

The scope of this work is to tackle this issue through the development and testing of a multiscale approach for simple polymer interfaces. Here, we consider Poly-Ether-Ether-Ketone (PEEK), a semicrystalline thermoplastic with outstanding mechanical properties, thermal stability, and chemical resistance, thus constituting an excellent material for bearing and sealing applications. Few MD studies exist for this material, mostly focused on its structural $[21,22]$ and mechanical properties [23,24], but not on its tribological performance. In this open field, we will investigate what insights MD can provide into friction at the molecular scale, and especially on the shear strength of adhesive junctions. For simplicity, impurities and fillers usually found in polymers are not considered in this article. Instead, a pristine PEEK-PEEK interface is modelled first, and interfacial water is added next to analyze the effect of physisorbed fluids. A parametric study on the loading conditions is also performed to assess the transferability of MD friction results to larger scales.

We will then bridge the gap between atomistics and continuum by integrating MD results into contact mechanics simulations to estimate the friction coefficient in macroscopic systems. Finally, tribometer experiments are performed and compared to the results of our multiscale approach.

\section{Methods}

Our multiscale methodology involves the study of two main scales. At the nanometer scale, atomistic simulations are employed to understand the details of tribological phenomena such as structural transformations in the polymers, and to formulate local friction laws for the relevant contact interfaces. At the macroscopic scale, the real area of contact between rough polymer surfaces is determined by using contact mechanics calculations. By applying the local friction laws to the real contact area, macroscopic friction coefficients can be estimated and compared to results from tribometer experiments. 
Note, that the polymer-polymer pairing in this work is less common in tribological applications than polymer-metal contacts. The simpler interface - especially with respect to atomistic simulations - allows for more straightforward testing of the proposed multiscale approach, which can then be applied to more complex and technically relevant systems.

\subsection{Atomistic modelling of bulk PEEK}

Non-reactive MD is employed to quantify nanoscale friction mechanisms at dry and moist PEEK-PEEK interfaces. This method involves integration of Newton's equations of motion for each atom in a system and provides its time evolution under tribological loading. Intra- and intermolecular interactions for the polymer are described by the OPLS-AA force field and in the BOSS/Tinker package [25,26], while the TIP3P potential [27] is employed for water molecules. All simulations are performed using the software package LAMMPS [28] with a time step of 1 femtosecond. OVITO [29] is used for the visualization of atomic trajectories.

As a first step, we benchmarked the OPLS-AA potential by modelling the structural and mechanical properties of bulk PEEK and by comparing the results to experimental data. For relaxed crystalline structures the lattice constants, characteristic angles and density were in good agreement with X-ray diffraction experiments [21], with a maximum difference of 5\% (Table 1).

\begin{tabular}{|c|c|c|}
\hline Property & Simulated & Experimental \\
\hline & \multicolumn{2}{|c|}{ Crystalline } \\
\hline \multirow{3}{*}{ Lattice constants $[\AA ̊]$} & $a=7.41$ & $a=7.75$ \\
\hline & $b=6.00$ & $\mathrm{~b}=5.89$ \\
\hline & $c=10.3$ & $c=9.88$ \\
\hline Ether C-O-C angle [ [ $\left.{ }^{\circ}\right]$ & 119 & Approx. 125 \\
\hline $\begin{array}{l}\text { Ketone } \quad \mathrm{C}-\mathrm{C}(=\mathrm{O})-\mathrm{C} \\
\text { angle }\left[{ }^{\circ}\right]\end{array}$ & 125 & Approx. 125 \\
\hline $\begin{array}{l}\text { Inclination of phenyl } \\
\text { ring }\left[^{\circ}\right]\end{array}$ & 34 & Approx. 37 \\
\hline Density $\left[\mathrm{g} / \mathrm{cm}^{3}\right]$ at $0 \mathrm{~K}$ & 1.40 & - \\
\hline \multirow[t]{2}{*}{ Density $\left[\mathrm{g} / \mathrm{cm}^{3}\right]$ at $298 \mathrm{~K}$} & 1.36 & 1.43 \\
\hline & \multicolumn{2}{|c|}{ Amorphous } \\
\hline \multirow[t]{2}{*}{ Density $\left[\mathrm{g} / \mathrm{cm}^{3}\right]$ at $298 \mathrm{~K}$} & $1.19 \mathrm{~g} / \mathrm{cm}^{3}$ & $1.26[30]$ \\
\hline & Amorphous PEEK, $T=0 K$ & Semicryst. PEEK, amb. T \\
\hline Young's modulus [GPa] & 5.2 & $3.6-4.1[31]$ \\
\hline Poisson's ratio [-] & 0.4 & $0.4[31]$ \\
\hline
\end{tabular}

Table 1: Structural and mechanical properties of the crystalline and amorphous bulk PEEK phases. Experimental data is from reference [21] otherwise noted.

Several amorphous bulk PEEK structures with different oligomer lengths are then generated. We consider oligomers with molecular weights ranging from 1000 to $9000 \mathrm{~g} / \mathrm{mol}$, corresponding to chains lengths from 4 up to 36 repeating units. Starting from low density and high temperature conditions $\left(0.1 \mathrm{~g} / \mathrm{cm}^{3}, 1000 \mathrm{~K}\right)$ to randomize molecular configurations, each system is compressed to reach a realistic density and quenched down to $0 \mathrm{~K}$ at a rate of 0.25 $\mathrm{K} / \mathrm{ps}$. Finally, relaxation at ambient pressure is performed to obtain stress-free amorphous structures. Elastic constants at $0 \mathrm{~K}$ are then computed by applying small strains (up to $0.5 \%$ ) to the relaxed structures and quantifying the resulting stresses [32]. The computed Young's modulus and Poisson's ratio agree well with experimental values [31], although the latter have been measured for semi-crystalline PEEK at ambient temperature (Table 1). 
In summary, these findings suggest that the OPLS-AA force field is suitable for a PEEK/PEEK friction study since it reproduces realistic structural and mechanical properties of amorphous and crystalline bulk PEEK systems. We also find that properties of the amorphous systems do not vary significantly for chains longer than 12 repeating units. Therefore, we employed bulk systems consisting of hexadecamers for our subsequent simulations.

\subsection{Atomistic simulations of amorphous PEEK-PEEK shear interfaces}

Amorphous PEEK surfaces are created from amorphous bulk structures by splitting in half while ensuring that no polymer chains are cut in the process. Relaxation at ambient temperature without additional constraints leads to the formation of nanoscale roughness as dangling polymer chains fold onto the surface (Figure 1a). Conversely, smooth surfaces or defined sinusoidal roughness (Figure $1 \mathrm{~b}-\mathrm{c}$ ) are obtained by reheating and quenching the surfaces under compression through artificial walls with the desired shape. Each surface block has a volume of $12 \times 8 \times 18 \mathrm{~nm}^{3}$ and contains 256 oligomers. We limit the scope of the molecular dynamics analysis to amorphous PEEK surfaces, as typical spherulitic crystalline structures in this polymer [33] possess amorphous regions with bent and dangling chains at their surface.

a)

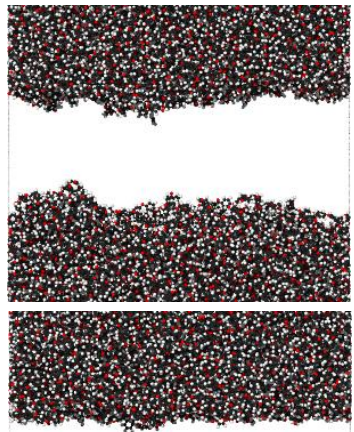

b)

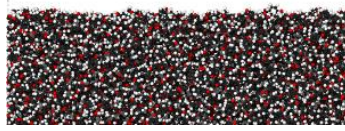

c)
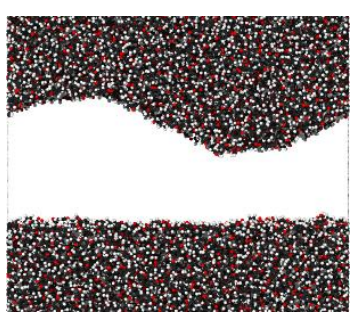

d)

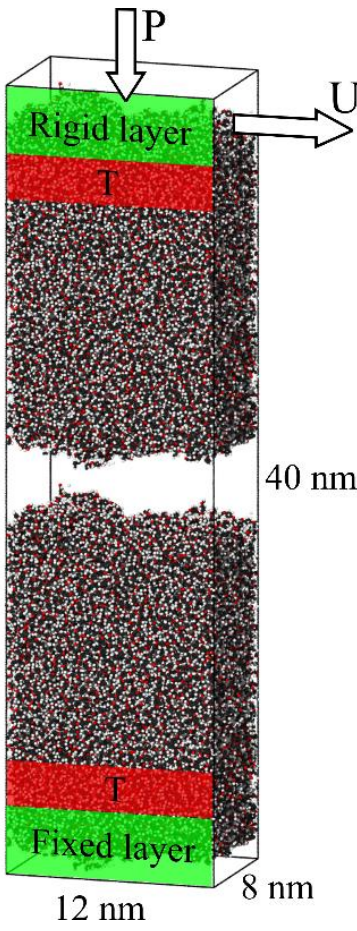

Figure 1: a-c) Amorphous PEEK surfaces: nanorough with $h_{R M S}=4.3 \AA$, smooth with $h_{R M S}=2.3 \AA$, and a sinusoidal geometry with $2 \mathrm{~nm}$ amplitude and $12 \mathrm{~nm}$ wavelength. d) Molecular dynamics sliding system.

Amorphous PEEK-PEEK tribological interfaces are simulated by pairing 2 surface blocks which are then subject to compression and shear. Periodic boundary conditions are applied along the system length and width. Each surface block is divided into three regions (Figure 1d): a $4 \mathrm{~nm}$-thick rigid layer where normal load and shear velocity are applied, a $1 \mathrm{~nm}$-thick thermostated domain with $T=300 \mathrm{~K}$, and a free unconstrained region around the shearing interface.

Applied external pressures for the compression vary in the range $\mathrm{P}=1-100 \mathrm{MPa}$. Shearing is then performed under constant normal load with a reference velocity of $U=100 \mathrm{~m} / \mathrm{s}$. This high value is due to the high computational cost of all-atom non-reactive MD simulations which 
limits realistic simulation times to a few nanoseconds. In our simulations, a sliding distance of $0.2 \mu \mathrm{m}$ (simulated time of $2 \mathrm{~ns}$ ) is typically required to reach steady-state and to quantify the shear response of the interface. Nonetheless, lower velocities of $2 \mathrm{~m} / \mathrm{s}$ and $10 \mathrm{~m} / \mathrm{s}$ are also considered to assess the speed dependence of friction processes.

Finally, two types of interfaces are simulated. In the "dry" case the contact occurs between pure PEEK surfaces. Conversely, water is added between rough PEEK surfaces in the "moist" case to investigate the influence of physisorbed liquids. Up to $1500 \mathrm{H}_{2} \mathrm{O}$ molecules are considered between the two $12 \times 8 \mathrm{~nm}^{2}$ surfaces, i.e. up to a surface density of $0.5 \mathrm{ng} / \mathrm{mm}^{2}$ representing a single water monolayer physisorbed on each PEEK surface.

\subsection{Contact mechanics simulations}

Elastoplastic contact mechanics calculations are performed to obtain the real contact area and pressure distribution in the rough ball-on-plate system used in the experiments. The calculations employ the code pyCo by L. Pastewka [34], based on an FFT-based Boundary Element Method $[35,36]$ together with a conjugate gradient optimization method [37] for fast convergence.

Representative surface topographies of the PEEK sphere and countersurface used in the calculations were obtained from AFM measurements of unworn experimental samples (Figure 2). The power spectral densities of all samples show a similar trend at high wavenumbers with a Hurst exponent of approximately 0.8. It should be noted that the topography data of the spheres also includes their macroscopic curvature, which explains the higher PSD at lower wavenumbers compared to the flat counter-surfaces.

a)

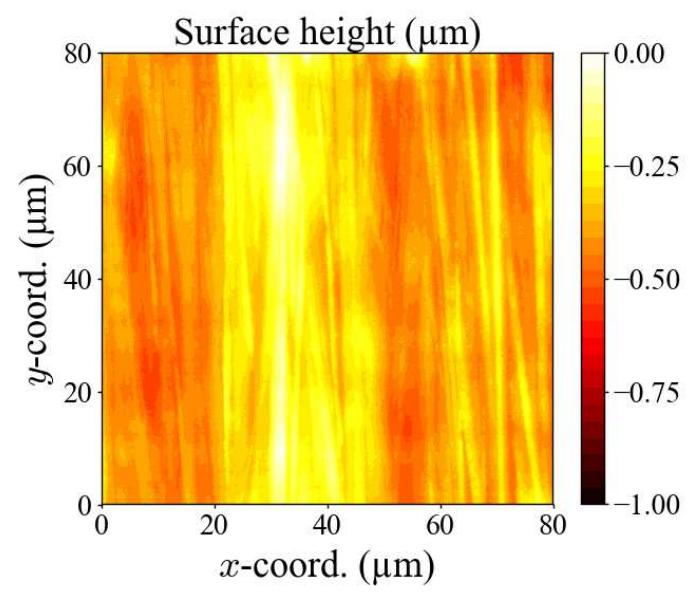

c)

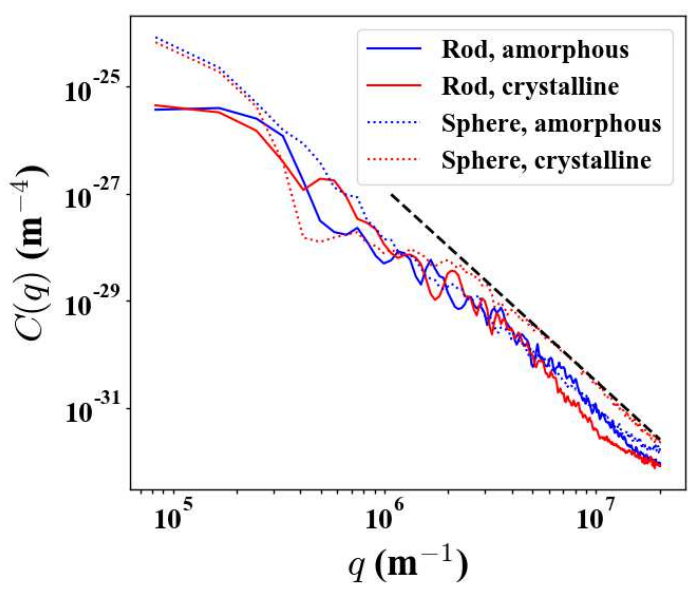

b)

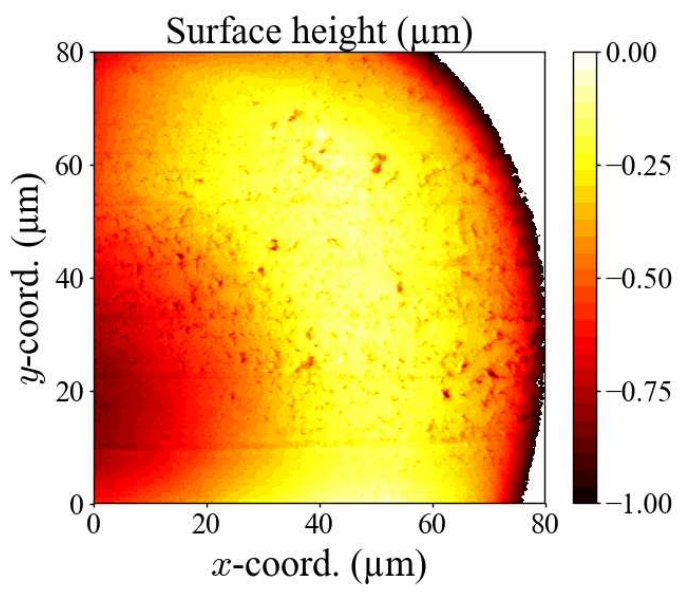

Figure 2: AFM scans of surface roughness of a) a traction rod, used as plane, and b) a sphere with $1.5 \mathrm{~mm}$ radius used in the tribometer experiments. The AFM resolution is $0.16 \mu \mathrm{m}$ in both directions. c) Radially averaged power spectral densities of the surface topographies. The dashed line indicates a Hurst exponent of $H=0.8$. 
In the contact mechanics code, the topographies of the sphere and countersurface are combined and pressed against a half space with equivalent elastic modulus $E^{*}=\frac{E_{P E E K}}{2\left(1-v^{2}\right)}$. Plasticity is included by limiting the contact pressure to the penetration hardness of the materials [3]. The material parameters used in the contact mechanics calculations were experimentally determined for PEEK samples used in this work and are summarized in Table 2.

Finally, the sliding motion of the surfaces is not simulated explicitly in this work. Instead, the ball is pressed against the countersurface at several locations along the sliding direction, so that the sliding is reduced to a series of quasi-static cases with pure normal loading.

\subsection{Experimental setup}

Neat PEEK samples with two different degrees of crystallinity were prepared in-house at Freudenberg Sealing Technologies. Differential calorimetry was used to assess the degree of crystallinity within the bulk thermoplastic: hereafter samples containing a $20 \%$ and $40 \%$ crystalline phase will be referred as "amorphous" and "crystalline" respectively.

AFM morphology measurements reveal the presence of flake-like structures ranging from 150 $\mathrm{nm}$ to $500 \mathrm{~nm}$ in length on the surfaces of both amorphous and crystalline samples. These domains are interpreted as crystalline regions on the surface, with a local surface coverage up to $\sim 25 \%$. However, due to the non-uniform distribution of the flake-like structures, it is difficult to extract the degree of crystallinity over the entire surfaces from the aforementioned measurements.

To further differentiate between amorphous and crystalline samples and extract their elastic modulus and hardness at the surface, nanoindentation are performed on a Hysitron TI950 device. A Berkovich tip is used on 10x10 indent grids, with two indentation depths of $0.3 \mu \mathrm{m}$ and $1.5 \mu \mathrm{m}$. The mechanical properties of the amorphous sample decrease with lower indentation depth. This is confirmed by indentation on a sample cross-section performed at different distances from the surface. Here, the thickness of the surface zone is found to be 0.4 $\mu \mathrm{m}$. A change in the mechanical properties with indentation depth is not observed on the crystalline samples. Instead, increased variation in elastic modulus and hardness across the indentation grid is found, which hints at a larger heterogeneity of the crystalline sample.

Averaged values for the mechanical properties are summarized in Table 2. Here, both the elastic modulus and hardness decrease significantly when going from the maximum crystalline content $(40 \%)$ of the "crystalline" sample to $20 \%$ of the "amorphous" one.

\begin{tabular}{|l|c|c|}
\hline Sample & $\begin{array}{c}\text { Amorphous } \\
(20 \% \text { crystallinity })\end{array}$ & $\begin{array}{c}\text { Crystalline } \\
(40 \% \text { crystallinity })\end{array}$ \\
\hline Young's modulus $\boldsymbol{E}$ [GPa] & $4.0 \pm 0.1$ & $5.2 \pm 0.4$ \\
\hline Indentation hardness [MPa] & $120 \pm 10$ & $260 \pm 40$ \\
\hline Poisson's ratio $\boldsymbol{v}[-]$ & 0.4 & 0.4 \\
\hline
\end{tabular}

Table 2: Material properties of neat PEEK samples determined through nanoindentation at an indentation depth of $0.3 \mu \mathrm{m}$. The Poisson's ratio is from ref. [31].

A BASALT-MUST ball-on-plate reciprocating tribometer by TETRA GmbH is used for the experimental analysis of PEEK-PEEK friction. In the tribometer, a sphere with radius of $\mathrm{R}=1.5$ $\mathrm{mm}$ is placed in contact with a tensile rod serving as the plane counter surface. Both samples were produced using the same molding tool. 
The operating conditions for the experiments are summarized in Table 3. The four loads correspond to Hertzian pressures ranging from 10 to $75 \mathrm{MPa}$ (the upper limit being close to the maximum operating limit of PEEK). Thus, a low sliding velocity of $2 \mathrm{~mm} / \mathrm{s}$ is chosen to avoid overloading and excessive heat generation at the contact interface. Breakaway forces at reversal points are excluded from the evaluation of the coefficient of friction.

\begin{tabular}{|l|c|}
\hline Geometry & Ball on Block \\
\hline Movement & Reversing \\
\hline Cycles & 2000 \\
\hline Load $(\mathbf{m N})$ & $6,130,310,1050$ \\
\hline Velocity (mm/s) & 2 \\
\hline Sliding distance (mm) & 10 \\
\hline
\end{tabular}

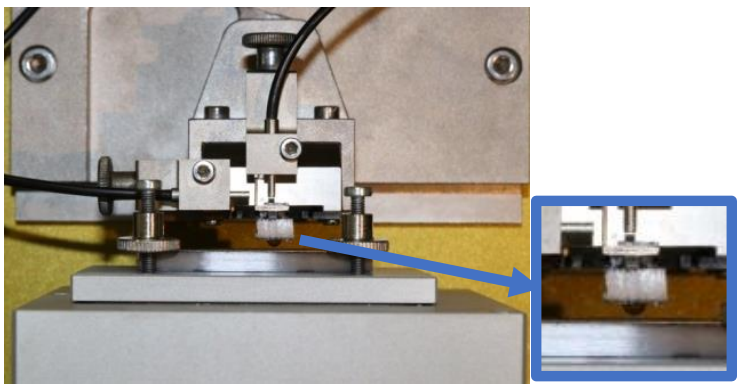

Table 3: Parameters for the tribometer friction experiment. Pictures of the apparatus and the sphere-rod contact are shown on the right.

\section{Results and discussion}

\subsection{Atomistic simulations of dry PEEK-PEEK interfaces}

As a first step in the multiscale approach, we aim at understanding the atomic-scale tribological processes occurring at dry PEEK-PEEK interfaces.

Figure 3 shows the evolution of the shear stress $\tau$ with the sliding distance for the three different interface geometries from Figure $1 \mathrm{a}-\mathrm{c}$. For all three simulations the loading conditions $P=100$ $\mathrm{MPa}, U=100 \mathrm{~m} / \mathrm{s}, T=300 \mathrm{~K}$ are chosen. At the beginning of shearing, $\tau$ increases linearly as the surfaces are elastically deformed over their entire height before the onset of localized sliding at the interface. For the nanorough surface, this occurs after a sliding distance of $5 \mathrm{~nm}$ corresponding to a stress peak of $120 \mathrm{MPa}$. Friction then decreases, and $\tau$ stabilizes to a value of $80 \mathrm{MPa}$ as steady state is reached.

The friction response of both the smooth-smooth and sinusoidal-smooth pairings is very similar to the nanorough one over the whole sliding distance. In order to explain these similarities in the friction response of the three interfaces, we look at transformations in terms of surface roughness and molecular configurations of the oligomer chains. The line root mean square height along the shearing direction is defined as:

$$
h_{\text {line }, R M S}=\frac{1}{n} \sum_{i=1}^{n} \sqrt{\frac{1}{L} \int_{0}^{L}\left[h\left(x, y_{i}\right)-\bar{h}\left(y_{i}\right)\right]^{2} d x}
$$

where the outer sum corresponds to averaging over the system width $\mathrm{W}, \mathrm{h}(x, y)$ represents the envelope of the surface atoms with a sphere of radius $1.4 \AA$ [38], and $\bar{h}(y)$ is the average line height in the $x$-direction.

Figure $3 b$ shows the evolution of the line RMS height with the sliding distance. After reaching steady state, the $h_{\text {line,RMS }}$ value of the nanorough surface is reduced by half relative to the starting configuration. The reduction in roughness under shearing is even more pronounced for 
the sinusoidal profile, which is completely flattened in less than $20 \mathrm{~nm}$ sliding distance. It should be noted that this phenomenon is favored at elevated pressures such as $\mathrm{P}=100 \mathrm{MPa}$, which is close to the penetration hardness of PEEK. Nonetheless, the same flattening process was also observed at lower pressures such as $10 \mathrm{MPa}$.

A peculiar behavior is observed for the smooth-smooth interface, whose friction evolution with the sliding distance follows the nanorough case very closely. While the initial surfaces are almost atomically flat, roughening at the interface occurs as soon as shearing is applied: $h_{\text {line } R M S}$ is doubled, then decreases slightly as steady state is reached. This behavior can be explained through the change in orientation of the oligomer chains at the interface as seen in Figure $3 \mathrm{c}$-d. Initially, the ends of the oligomer chains in the interfacial region are oriented randomly but tend to align along the sliding direction as soon as shearing is applied. This reconfiguration process results in significant roughening of the initially atomically flat interface, leading to $h_{\text {line,rms }}$ and shear stress similar to the nanorough case.

a)

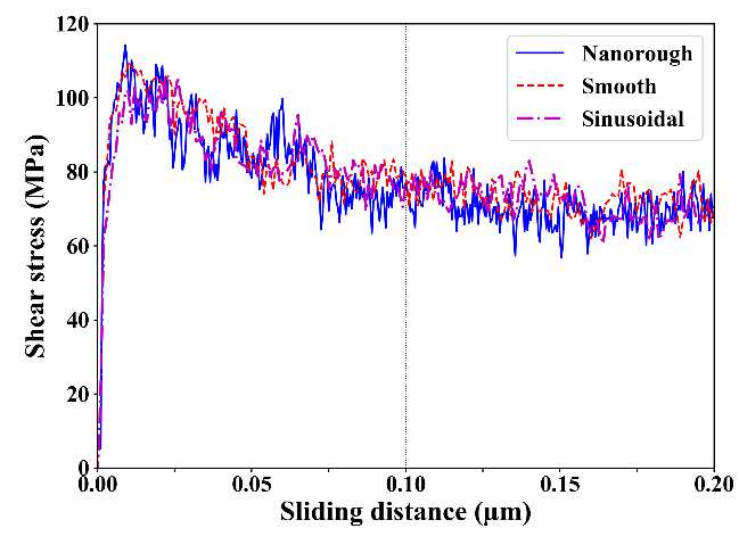

c)

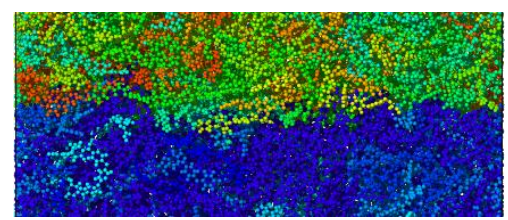

b)

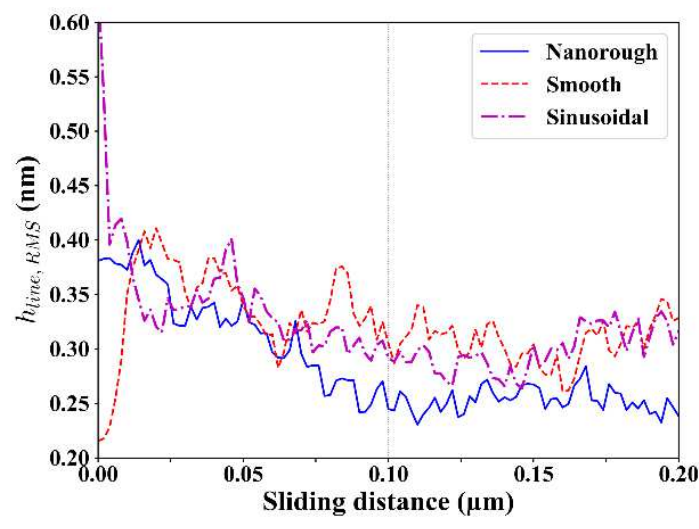

d)

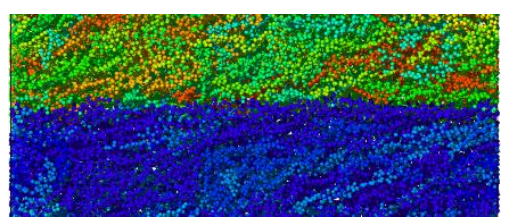

Figure 3: a) Shear stress and b) line RMS roughness for three amorphous PEEK-PEEK interfaces as a function of the sliding distance $(P=100 \mathrm{MPa}, U=100 \mathrm{~m} / \mathrm{s}, T=300 \mathrm{~K})$. After $0.1 \mu \mathrm{m}$ sliding, steady state friction is reached. c) Snapshot of the nanorough interface at the beginning of shearing and d) after $0.1 \mu \mathrm{m}$ sliding distance. Different colors indicate different oligomer chains (top surface red to green, bottom surface cyan to blue).

We quantify chain alignment by analyzing a simplified representation of the PEEK molecules (Figure 4a). From the all-atom representation, segments linking the Ether-Oxygens and the Ketone-Carbon atoms are extracted. Each segment is characterized by its center point and an angle $\theta$ with respect to the shearing direction $\vec{x}$. While the distribution of $\theta$ at the PEEK-PEEK interface prior to sliding is uniform as expected for an isotropic amorphous polymer material (blue curve in Figure 4b), after steady-state sliding is reached a strong alignment along the shearing direction can be observed (red curve in Figure 4b). Next, we analyze the angle distribution of PEEK chains in the vicinity of the sliding interface in more detail. To do so, we chose as criterion for aligned segments that their angle with the shearing direction must be lower than a certain cutoff value (here $|\theta|<\pi / 4$, see Figure $4 b-c)$. Figure $4 d$ shows the percentage of aligned segments along the system height before shearing and once steady state friction is reached. All initial configurations show approximately $29 \%$ of aligned segments over 
the whole system height, corresponding to chains without preferential orientation as explained in Figure 4c. During sliding the oligomer chains within a $\pm 3 \mathrm{~nm}$ proximity of the sliding interface align along the shearing direction. This process occurs over a sliding distance of 0.1 $\mu \mathrm{m}$, which is also required to reach steady state friction. Afterwards, no growth of the structurally aligned region is detected, although this may be due to the sliding distance being limited to $0.2 \mu \mathrm{m}$ in our molecular dynamics simulations.

a)

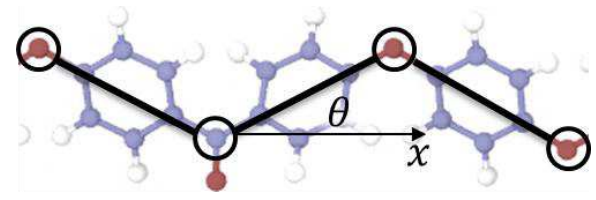

b)

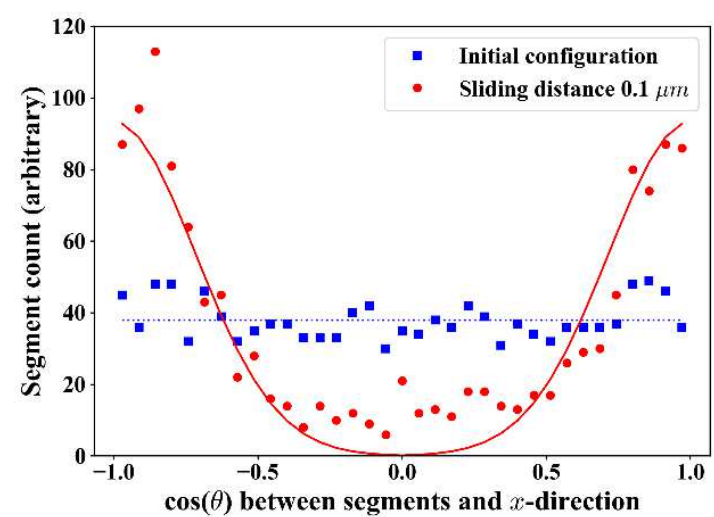

c)

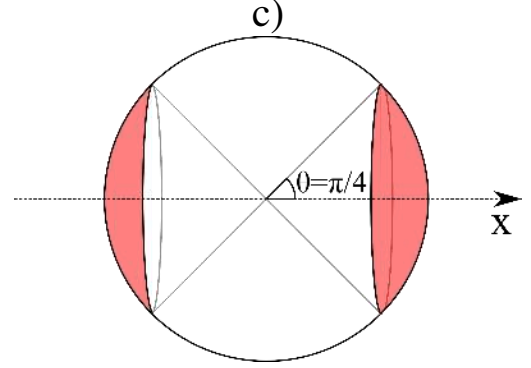

d)

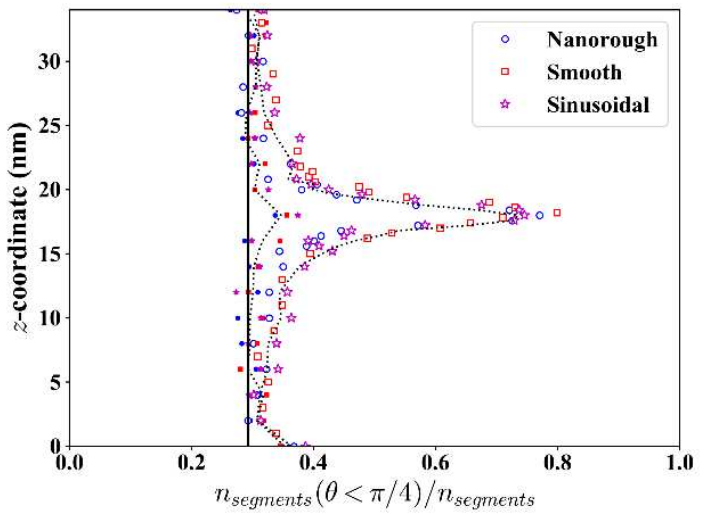

Figure 4: a) Representation of simplified PEEK chain used in the analysis. b) Segment angle distribution at the sliding interface. The continuous red line is a Gaussian curve with standard deviation corresponding to $|\theta|=\pi / 4$. c) Aligned segments are contained within two spherical sectors featuring an aperture angle of $\pi / 4$ with respect to the $x$-direction. In systems without preferential orientation, the percentage of the aligned is given by the surface of the two spherical sectors, divided by the sphere surface. This gives $1-\cos (\pi / 4) \approx 0.29$. d) Number of aligned segments over the system height, with the sliding interface being located at $z=18 \mathrm{~nm}$. Full markers correspond to the beginning of shearing, whereas empty markers indicate steady state shear results.

Next, we quantify friction in the PEEK-PEEK systems as a function of the loading conditions. First, the external pressure is varied from $\mathrm{P}=1$ to $100 \mathrm{MPa}$. For the considered interfaces, the shear stress in steady-state sliding is generally high and increases linearly with the applied pressure (Figure 5). The slope of a linear fit to the $\tau(\mathrm{P})$ curve is interpreted as the atomic scale friction coefficient $\mu_{M D}$. Interestingly, $\mu_{M D}$ is approximately 0.2 irrespective of the initial roughness of the PEEK surfaces. Furthermore, strong adhesion between the PEEK surfaces occurs due to their chemical affinity, the absence of interposed media, and full contact at the sliding interface, resulting in a large shear stress offset $\bar{\tau}_{a d h}$. This adhesive contribution to friction is of the order of the bulk shear strength of PEEK, akin to what was found for polystyrene through experiments in [3]. 


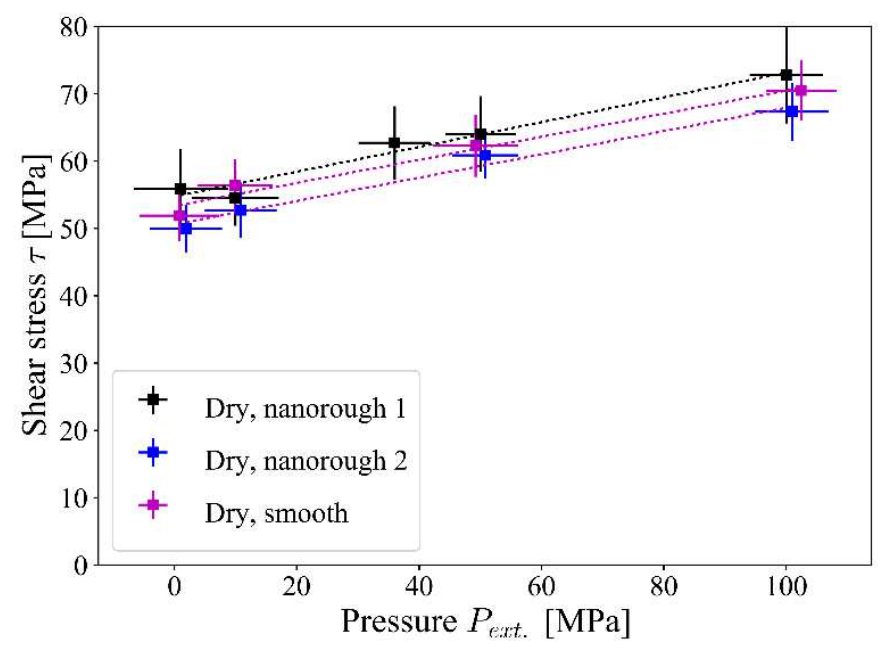

Figure 5: Shear stress in dry amorphous PEEK-PEEK interfaces as a function of the applied pressure $(U=100 \mathrm{~m} / \mathrm{s}, T=300 \mathrm{~K})$. The dotted lines represent linear fits of the data points. Nanorough 2 represents an additional realization of a nanorough dry interface, obtained by removing water from one of the systems in Section 3.2.

Based on the similar behavior exhibited by all interfaces considered here, a single law can be employed to quantify the steady-state friction response as a function of the applied pressure:

$$
\bar{\tau}_{M D}(P)=\bar{\mu}_{M D} \mathrm{P}+\bar{\tau}_{a d h .}=0.18 \mathrm{P}+50.5 \mathrm{MPa}
$$

where ${ }^{-}$represents the average of the $\tau(P)$ curves for different interfaces.

Finally, the sliding velocity is reduced from $U=100 \mathrm{~m} / \mathrm{s}$ to $U=10 \mathrm{~m} / \mathrm{s}$ and $2 \mathrm{~m} / \mathrm{s}$ at $P=1 \mathrm{MPa}$ to investigate the polymer response under loading conditions usually found in technical systems. Figure 6a shows that for very high and low velocities, the shear stress only differs by $10 \%$ at the stress peak in the transient regime, and by only about $7 \%$ in the steady state regime. Additionally, as shown in Figure 6b, the structural transformations at the PEEK-PEEK interface depend only on the sliding distance. These results indicate that the shear stress response is almost independent on the shear velocity for the considered interfaces. Thus, the nanoscale friction law in Eq. 2 derived from simulations at $U=100 \mathrm{~m} / \mathrm{s}$ will be directly used for the calculation of macroscopic friction and the comparison with tribometer experiments, which were performed at velocities lower by 5 orders of magnitude.

a)

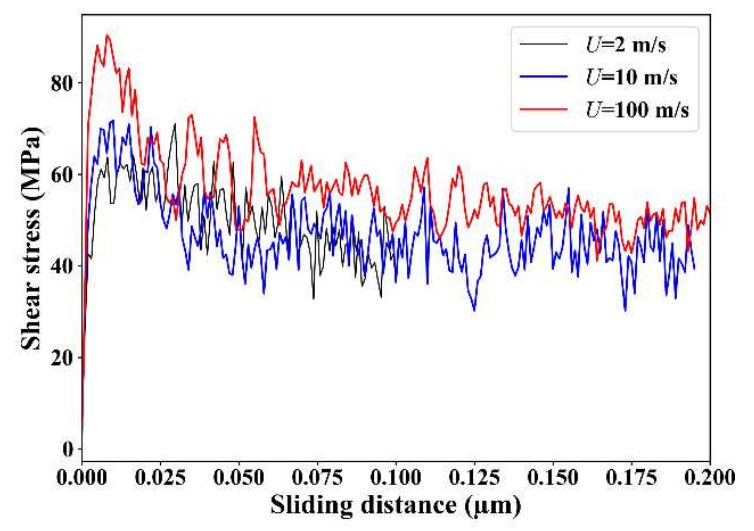

b)

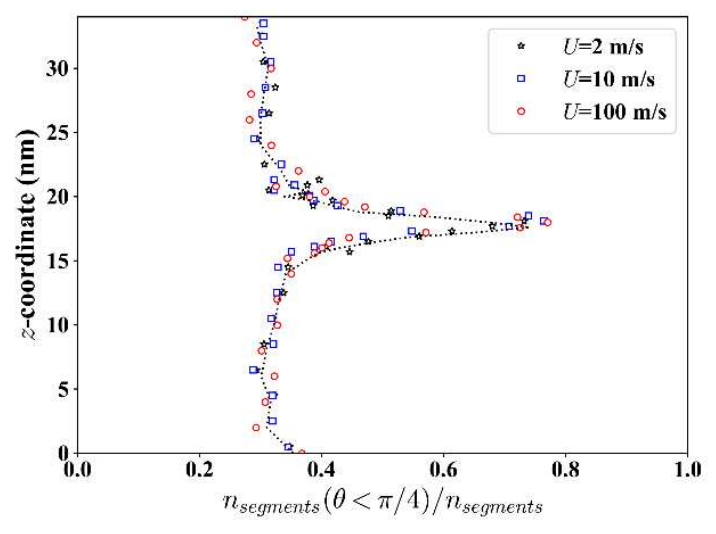

Figure 6: a) Shear stress as a function of the sliding distance at different sliding velocities and low load $(P=1 \mathrm{MPa}, T=300 \mathrm{~K})$. b) Chain alignment after a sliding distance of $0.1 \mu \mathrm{m}$ for three shear velocities. 


\subsection{Atomistic simulations of moist contact interfaces}

One should keep in mind that the shearing of pure polymer-polymer interfaces in the previous section only provides a very simplified representation of reality. This special situation could be characteristic of wear processes that rub away the initial surfaces and expose pure PEEK material at the sliding interface. Still, unworn samples generally exhibit an injection skin with modified crystallinity and material composition at their surface. Contaminants, impurities, or fluid molecules can also be found on top of the surfaces. Our previous results indicate that shear processes are strongly linked to the structure and topography in a nanometer-thin zone at the sliding interface. Thus, adhesion and friction can be strongly modified even by a small amount of physiosorbed fluid.

As a first attempt to investigate a more realistic sliding interface, $\mathrm{H}_{2} \mathrm{O}$ is inserted between the two amorphous surfaces with a surface density below one nanogram per $\mathrm{mm}^{2}$. This aims at modelling physisorption of water from air humidity [39], which is likely to occur onto the ketone and ether groups of the PEEK chains. Shearing simulations of water-lubricated PEEKPEEK interfaces are performed with the same pressures and sliding speed as in Figure 5. Under tribological loading, the water molecules help to separate the surfaces by forming nanochannels aligned along the sliding direction, thereby significantly reducing friction (Figure 7). Under shearing, small amounts of water penetrate the surface-near region of PEEK, but the present simulations are too short to evaluate diffusion of $\mathrm{H}_{2} \mathrm{O}$ within the polymer bulk. It should also be noted that water squeeze-out is not possible here, due to the lateral periodic boundary conditions of the Molecular Dynamics model.

Water between the surfaces acts as a lubricant and reduces interfacial friction due to the partial separation of the PEEK surfaces. Results in Figure 8 show a linear dependence between applied pressure and shear stress. The slope of the $\tau(\mathrm{P})$ line, i.e. the friction coefficient from $\mathrm{MD}$, is $\mu_{M D}=0.18$ as in the dry case. However, the offset $\bar{\tau}_{a d h}$. is significantly lower due to the smaller domains of direct polymer-polymer contact where intersurface adhesion takes place (Figure $5 b)$. This decrease can be approximated by a linear fit:

$$
\bar{\tau}_{a d h .}=50.5 \mathrm{MPa}-1.25 \sigma_{\mathrm{H}_{2} \mathrm{O}} \quad \text { Eq. } 3
$$

where $\sigma_{\mathrm{H}_{2} \mathrm{O}}$ is the surface density of water molecules in [number $/ \mathrm{nm}^{2}$ ] units. It should be noted that, although Eq. 3 reasonably describes the adhesive stress in presence of single water monolayers on pristine PEEK, it is eventually expected to break down as the amount of water at the interface is increased. In this case the surfaces become fully separated, and the rheology of the nanoconfined liquid and nano-hydrodynamics become the determining factors for the shear stress under sliding. 
a)

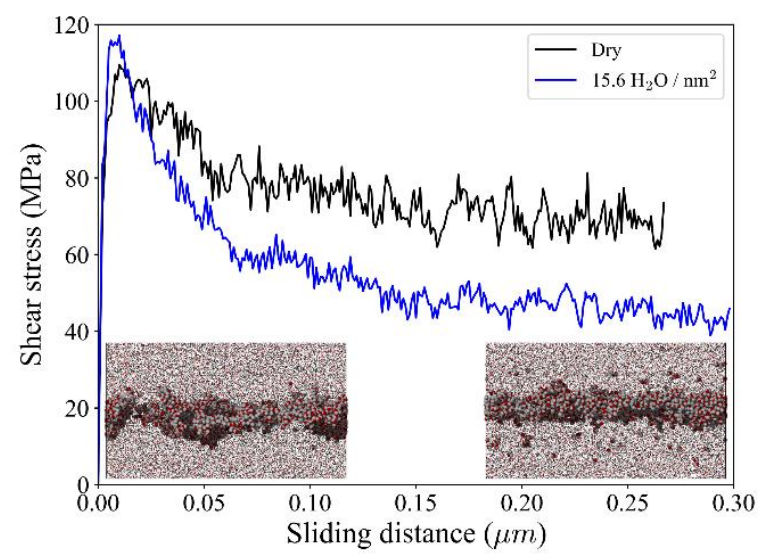

b)

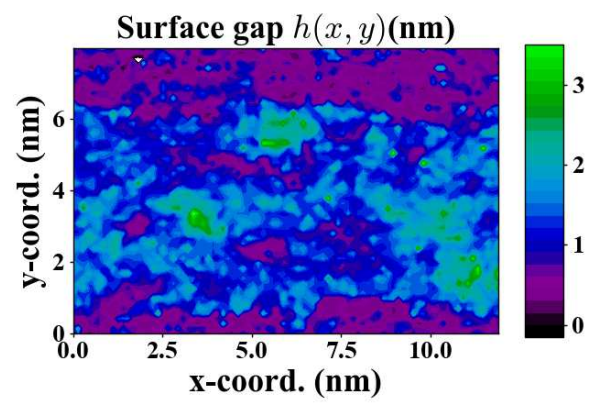

Figure 7: Shear stress as function of the sliding distance for a water-lubricated interface $(P=100 \mathrm{MPa}$, $U=100 \mathrm{~m} / \mathrm{s}, T=300 \mathrm{~K}, 15.6 \mathrm{n}_{\mathrm{H} 2 \mathrm{O}} / \mathrm{nm}^{2}$ ). The curve for a dry case under the same conditions is also shown for comparison. The insets depict the repartition of the water molecules at the sliding interface before sliding and after steady state is reached. b) Intersurface gap in steady state shearing. Purple domains correspond to PEEK-PEEK contact, whereas blue and green areas indicate a water nanochannel aligned in the shearing direction.

a)

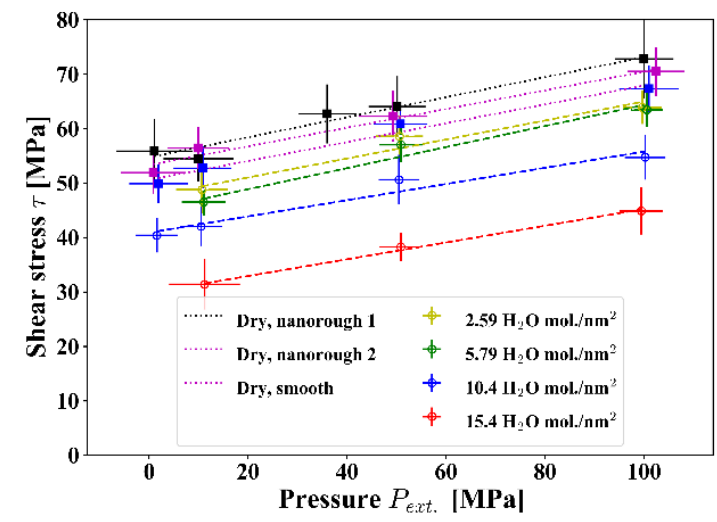

b)

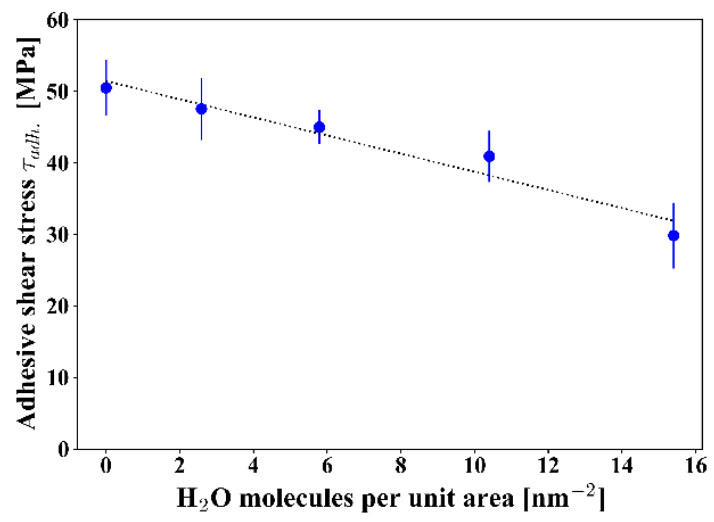

Figure 8: a) Shear stress in dry and water-lubricated PEEK-PEEK interfaces as a function of the applied pressure $(U=100 \mathrm{~m} / \mathrm{s}, T=300 \mathrm{~K})$. b) Dependence of the adhesive stress $\overline{\mathbf{\tau}}_{a d h}$. on the amount of water confined between PEEK surfaces. The dotted lines represent linear fits to the data points.

\subsection{Contact mechanics simulations and coupling with molecular dynamics}

In this section, the friction laws obtained from MD are coupled with continuum contact mechanics simulations to obtain a macroscopic friction coefficient. We assume that the $\bar{\tau}_{M D}(P)$ friction law from Eq. 2 applies to the contact domains between PEEK surfaces with microscopic roughness. The tangential force in the macroscopic contact is then obtained by integrating $\bar{\tau}_{M D}$ over the real area of contact $A_{\text {real }}$ :

$$
F_{T}=\iint_{A_{\text {real }}}\left[\bar{\mu}_{M D} P(x, y)+\bar{\tau}_{\text {adh. }}\right] d x d y
$$

where $P(x, y)$ is the local contact pressure. As shown in Section 3.1, the coefficients $\bar{\mu}_{M D}$ and $\bar{\tau}_{a d h .}$ are constant for all considered dry interfaces, leading to: 


$$
F_{T}=\bar{\mu}_{M D} \iint_{A_{c, r e a l}} P(x, y) d x d y+\bar{\tau}_{a d h} A_{\text {real }}
$$

The normal force $F_{N}$ is given by:

$$
F_{N}=A_{0} P_{\text {ext. }}=\iint_{A_{\text {real }}} P(x, y) d x d y
$$

with $A_{0}$ being the apparent contact area and $P_{\text {ext. }}$ the average normal pressure applied to the system.

Finally, the ratio $F_{T} / F_{N}$ gives the macroscopic friction coefficient:

$$
\mu_{\text {macro }}=\bar{\mu}_{M D}+\frac{\bar{\tau}_{\text {adh. }} .}{P_{\text {ext. }}} \frac{A_{\text {real }}}{A_{0}}
$$

The term $\frac{A_{\text {real }}}{A_{0}}\left(P_{\text {ext. }}\right)$ is determined from our contact mechanics simulations of real rough contact geometries obtained from AFM scans with elastoplastic material properties from Table 2. The relative contact area is computed for multiple sample topographies, i.e. for one ball and two rods for both the crystalline and amorphous samples, for several relative positionings of the surfaces, and for external pressures $P_{\text {ext }}$. ranging from 0.1 to $50 \mathrm{MPa}$, corresponding to loads up to $0.3 \mathrm{~N}$. The resulting values of $A_{\text {real }}$ do not depend significantly on the exact surface topography. This can be traced back to the similarity among the power spectra for all considered topographies, especially at high wavenumbers (Figure 2). Additionally, the contact pressures are limited by the indentation hardness of PEEK, which further reduces the contact area dependence on local topographical features. Finally, it should also be noted that accounting for plasticity significantly increases the contact area compared to purely elastic simulation, akin to [3].

Figure 9 shows the relative contact area at different external loads, where each point corresponds to the average value over all considered surface topographies and relative positions of the rod-sphere pairings. A linear dependence is found, in agreement with results from reference [3]. The slopes for both the crystalline and amorphous materials are $\frac{A_{\text {real }}}{A_{0} P_{\text {ext. }}}=$ $6.56 \square 10^{-3} \mathrm{MPa}^{-1}$ and $1.03 \cdot 10^{-2} \mathrm{MPa}^{-1}$, respectively. The difference in values is mainly due to the significantly higher penetration hardness of crystalline PEEK, and, to a more limited extent, to its larger Young's modulus. 


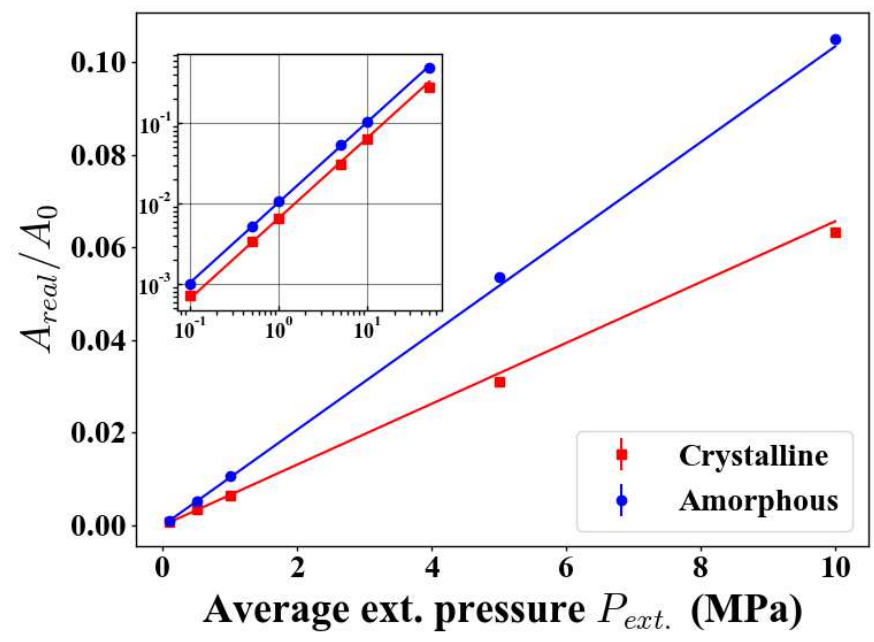

Figure 9: Relative contact area as a function of the external pressure. The inset features the same data with logarithmic axes. Both straight lines in the inset have a slope of 1, indicating linearity between relative contact area and applied load over several orders of magnitude. The external pressure up to $50 \mathrm{MPa}$ (inset) over the considered surface area corresponds to a normal load up to $0.32 \mathrm{~N}$.

Finally, Eq. 2 and the ratios $\frac{A_{\text {real }}}{A_{0} P_{\text {ext }}}$ from contact mechanics are used in Eq. 7 to estimate the friction coefficient of dry PEEK-PEEK interfaces. We obtain $\mu_{\text {macro }}=0.68$ and $\mu_{\text {macro }}=0.50$ for the amorphous and crystalline pairings, respectively. The higher friction coefficient in dry conditions for the amorphous case originates in the softer material properties which lead to a larger contact area at all loads.

In presence of small amounts of water at the interface, friction is reduced due to the lower adhesion offset $\bar{\tau}_{a d h}$. between polymer surfaces (Figure 8). For instance, with the maximum water quantity simulated in one of the previous sections, one finds $\mu_{\text {macro }}=0.28$. The reduction in friction due to the presence of water agrees qualitatively with previous experimental results [9]. Note that our calculated $\mu_{\text {macro }}$ reflects only changes in friction at the nanometer scale, while modifications of mechanical bulk properties due to absorbed fluids within the polymer [10] are not accounted for. Finally, if adhesion is fully suppressed, one obtains $\mu_{\text {macro }}=$ $\bar{\mu}_{M D}=0.18$. In the following section, these results are compared to tribometer experiments.

\subsection{Experimental results and comparison with simulation}

Figure 10 shows the time evolution of dry friction for experiments at different loads for both amorphous and crystalline surface pairings. Two possible outcomes are observed, the first being a low friction coefficient $\mu_{\text {exp }} \approx 0.2$ which remains stable for the entire duration of the experiment. The second shows high friction $\left(\mu_{\text {exp }} \approx 0.45-0.65\right)$ and fluctuating values with the number of reciprocating cycles.

At the lowest applied load of $6 \mathrm{mN}$ low friction outcomes were predominant. For normal forces of $130 \mathrm{mN}, 310 \mathrm{mN}$ and $1.05 \mathrm{~N}$, high friction was observed in approximately half of the performed experiments, as the applied load approached the mechanical limit of the thermoplastic. Additionally, in the high friction cases a value of $\mu_{\text {exp }}=0.65 \pm 0.06$ was observed for the amorphous pairing ( $20 \%$ crystallinity), which is generally higher than $\mu_{\text {exp }} \approx 0.51 \pm 0.11$ found for PEEK with maximum crystalline content of $40 \%$.

Finally, the two friction outcomes of the experiments correlate with the occurrence of wear. Stable, low friction runs showed only a slight flattening of the sphere sample. Conversely, 
massive abrasive wear occurs at high $\mu_{\text {exp }}$, as material is transferred between the sphere and a wear track on the rod (Figure 10c). The wear process is likely related to the large temporal fluctuations in the friction coefficient, as material detachment requires localized shearing of the bulk polymer as well as transient changes in surface topography. Interestingly, "crystalline" samples feature larger variations of $\mu_{\text {exp }}$ with time compared to the "amorphous" ones, indicating that wear events could be linked to the removal of crystalline spherulites from the surfaces.

Next, we compare the results of the multiscale approach with our tribometer results. The calculated friction coefficients for dry PEEK-PEEK interfaces $\left(\mu_{\text {macro }}=0.68\right.$ and 0.50 for the amorphous and crystalline pairings) are in good agreement with the high friction states observed in the experiments $\left(\mu_{\text {exp }}=0.65\right.$ and $\left.\mu_{\text {exp }}=0.51\right)$ - see Figure 10a,b . As explained in the previous section, the differences in friction between different degrees of crystallinity can be traced back to the elastoplastic material properties of the PEEK samples: the softer the material, the higher the contact area and friction will be.

a) Amorphous PEEK pairing

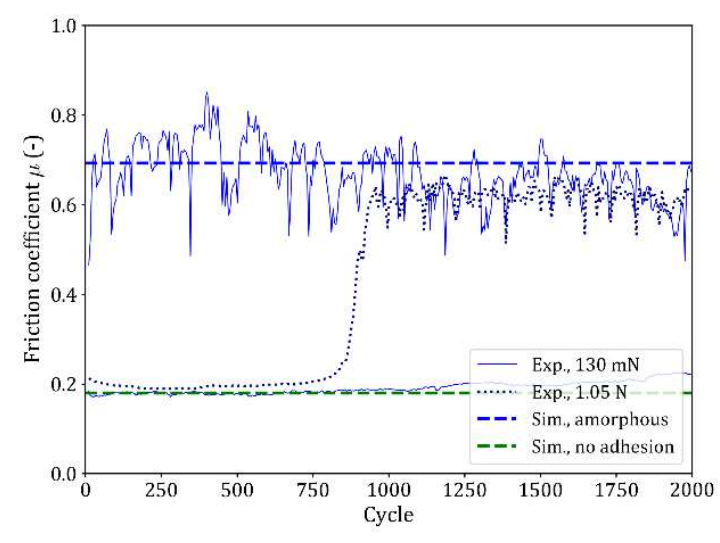

c)

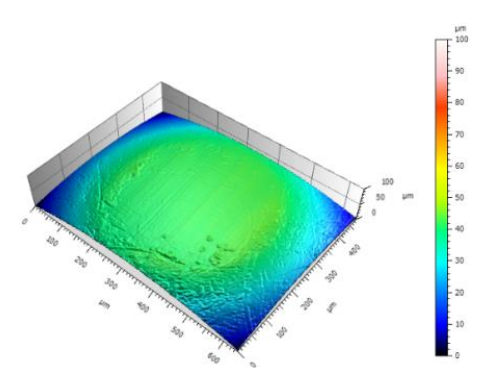

b) Crystalline PEEK pairing
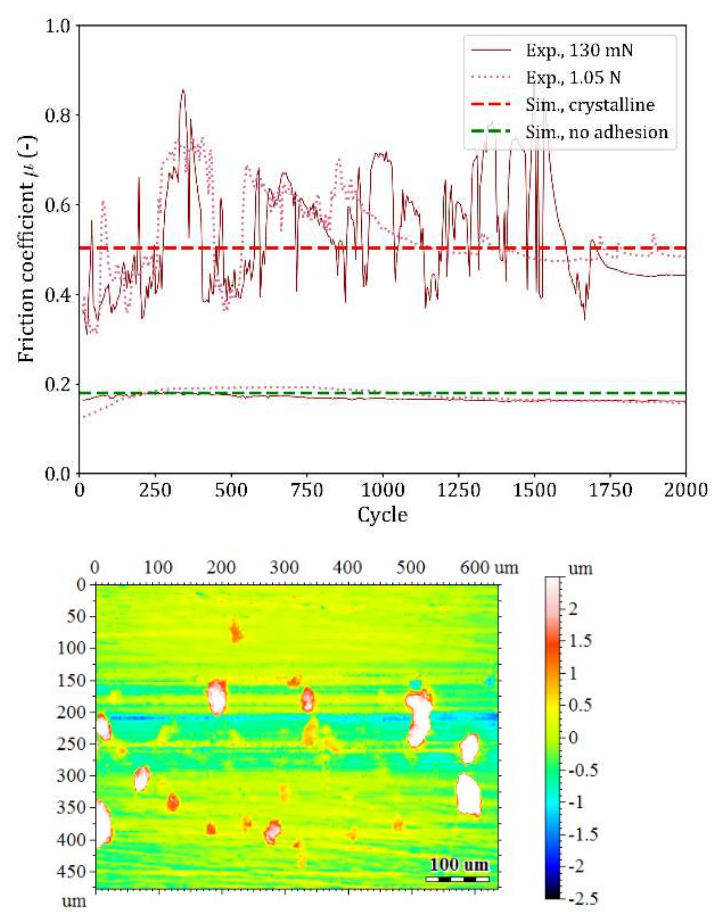

Figure 10: a-b) Experimental friction coefficient as a function of the number of reciprocating cycles for the amorphous and crystalline pairings, respectively. c) Confocal microscope image of a worn crystalline sphere and material transfer to the rod after a high friction run at $0.310 \mathrm{~N}$ load.

The good agreement between simulations and experiments in high friction runs hints that, under loading conditions close to the mechanical limit of the polymer, the simplifications performed in our multi-scale approach are justified. For instance, recent work on semi-crystalline polymers shows that the roughness spectra at high wavenumbers remains largely unchanged after plastic deformation occurs [40], indicating that changes in surface topography during sliding may not need to be considered in detail for the calculation of the contact area and macroscopic friction. Thus, AFM topography data from unworn samples used in our contact mechanics calculation should suffice. Instead, accounting for mechanical properties e.g. hardness of different samples is crucial to correctly quantify friction and to explain the lower values consistently observed for the crystalline pairing compared to the amorphous one at high loads. Strain hardening under shearing, which would lead to lower contact area and friction and may explain the slight 
decrease in friction at long sliding distances, is not accounted for in our simulations as it appears to have only a minor impact on $\mu$.

Finally, the nanoscale friction law used in our multiscale approach was obtained for the sliding of pure PEEK-PEEK. Although this ideal interface appears to be a strong simplification compared to real systems, it is expected to occur as bulk polymer material is sheared and removed during wear processes characteristic of high friction states from tribometer tests. The results also suggest that our choice to limit the scope of the atomistic modeling to amorphous interfaces is justified, as hard crystalline spherulites are unlikely to be sheared while sliding is localized within the weaker amorphous phase.

It should be noted that our approach does not directly predict low frictions values occurring in approximately half of the experiments. The reason can be pinpointed to the fact that the simulated pure PEEK-PEEK interface is not fully representative of the initial molding skin, which is preserved under sliding in the absence of wear. For more accurate atomistic simulations of the initial interface, further knowledge on the chemical composition of the last nanometers of PEEK surfaces is required.

Another aspect which must be analyzed is the presence of physisorbed molecules from the environment at the tribointerface. In this work water was considered as a first example, showing that its presence in extremely small quantities can significantly reduce adhesion between pure PEEK surfaces. Interestingly, if the adhesion-related shear stress component is fully suppressed from the nanoscale friction law, one obtains a macroscopic friction coefficient $\mu_{\text {macro }}=\mu_{M D}$ in very good agreement with the low friction experimental runs. Further work is therefore required to better understand and generalize this promising result, especially on the hydrodynamics and squeeze-out of fluids, such as water or other lubricants, both at the nanometer and macroscopic contact scales.

\section{Conclusions}

In this letter we employ a multiscale simulation method to investigate friction of neat PEEKPEEK polymer interfaces and compare its results to tribometer experiments. As a first step in the modelling approach, non-reactive Molecular Dynamics simulations are considered. Surfaces with different roughness are cut from nanometer-sized amorphous PEEK blocks with realistic material properties. The surfaces are then paired together and sheared under different normal loads. Tribological processes at the nanoscale involve flattening of nanoscale roughness occurring in a transient regime, and strong alignment of PEEK chains along the sliding direction in the surface-near region.

The steady-state shear stress of the dry PEEK-PEEK interface is high due to the strong adhesion between the chemically affine pure surfaces. Steady state-friction increases linearly with the applied pressure and does not depend significantly on the sliding speed nor the initial geometry of the amorphous surface. This dependence is summarized in a nanoscale friction law.

As a first test of the impact of pyhsisorbed fluids on friction, water monolayers are considered in-between the surfaces. Their presence reduces the adhesion component of the shear stress, as the surfaces were separated through water nano-channels aligned in the shearing direction.

In the second step of our multiscale approach, the nanoscale friction law is coupled with elastoplastic contact mechanics simulations based on AFM surface topography and material 
properties from nanoindentation experiments. Here, the real area of contact is determined at several loads for samples with $40 \%$ and $20 \%$ crystallinity, the latter material showing larger contact due to its lower penetration hardness. Finally, the nanoscale friction laws are integrated over the calculated contact area, giving friction coefficients of $\mu_{\text {macro }} \approx 0.5$ and $\mu_{\text {macro }} \approx 0.7$ for the aforementioned PEEK samples in dry conditions. These results are compared with reciprocating tribometer experiments on a ball-on-plate geometry. Excellent agreement is found for high friction cases occurring at high loads close to the operating range of PEEK. Under these conditions inter-surface adhesion is high, massive wear occurs and the shearing of bulk polymer is expected during the experiments.

Low friction cases with $\mu_{\text {exp }} \approx 0.2$ and no signs of wear were also observed in the experiments, especially at low loads. Although these are not directly reproducible by the multiscale simulations (assuming completely dry contacts), the present results hint at a reduced intersurface adhesion e.g. through physisorbed fluids. We show as an example that water monolayers can reduce the adhesion component of friction by half. If adhesion is completely suppressed in the calculation of macroscopic friction, $\mu_{\text {macro }} \approx 0.2$ is predicted, which corresponds to the value measured during the low friction states. Thus, further work could aim at a more detailed analysis and modeling of surface chemistry, especially regarding the role of lubricants, contaminants, or the polymer molding skin.

Finally, the present multiscale method could be applied to technically relevant surface pairings, such as polymer-metal interfaces, in order to understand the mechanisms and structural transformations leading to transfer film formation and its consequences on friction.

\section{Acknowledgements}

The authors gratefully acknowledge the Gauss Centre for Supercomputing e.V. (www.gausscentre.eu) for providing computing time under project hfr14 through the John von Neumann Institute for Computing (NIC) on the GCS Supercomputer JUWELS at Jülich Supercomputing Centre (JSC). We also gratefully acknowledge the computing time for supporting calculations granted under project ASTC on the supercomputer ForHLRII funded by the Ministry of Science, Research and the Arts Baden-Württemberg and by the Federal Ministry of Education and Research. The authors also thank Freudenberg Sealing Technologies for the preparation of the polymer samples.

\section{Declarations}

The authors have no relevant financial or non-financial interests to disclose.

\section{References}

1. Tiwari, A., Miyashita, N. et al., Rubber friction: The contribution from the area of real contact. The Journal of Chemical Physics 148, 224701 (2018).

2. Bahadur, S. \& Ludema, K. C., The viscoelastic nature of the sliding friction of polyethylene, polypropylene and copolymers. Wear 18, 109-128 (1971).

3. Weber, B., Suhina, T. et al., Molecular probes reveal deviations from Amontons' law in multi-asperity frictional contacts. Nature Communications 9, 888 (2018).

4. Hyun, S., Pei, L. et al., Finite-element analysis of contact between elastic self-affine surfaces. Phys. Rev. E 70, 026117 (2004). 
5. Amuzu, J. K. A., Briscoe, B. J. \& Tabor, D., Friction and Shear Strength of Polymers. A S L E Transactions 20, 354-358 (1977).

6. Benabdallah, S. H., Static shear strength and adhesion friction of some thermoplastics. Polymer Engineering \& Science 33, 543-548 (1993).

7. Persson, B. N. J. \& Volokitin, A. I., Rubber friction on smooth surfaces. The European Physical Journal E 21, 69-80 (2006).

8. Singh, A. K. \& Juvekar, V. A., Steady dynamic friction at elastomer-hard solid interface: A model based on population balance of bonds. Soft Matter 7 (22), 10601-10611 (2011).

9. Unal, H. \& Mimaroglu, A., Friction and Wear Characteristics of PEEK and its Composite under Water Lubrication. Journal of Reinforced Plastics and Composites 25, 1659-1667 (2006).

10. Voyer, J., Klien, S. et al., Static and Dynamic Friction of Pure and Friction-Modified PA6 Polymers in Contact with Steel Surfaces: Influence of Surface Roughness and Environmental Conditions. Lubricants 7, 17 (2019).

11. Laux, K. A., Jean-Fulcrand, A. et al., The influence of surface properties on sliding contact temperature and friction for polyetheretherketone (PEEK). Polymer 103, 397-404 (2016).

12. Barry, P. R., Chiu, P. Y. et al., The effect of normal load on polytetrafluoroethylene tribology. Journal of Physics: Condensed Matter 21, 144201 (2009).

13. Chiu, P. Y., Barry, P. R. et al., Influence of the Molecular Level Structure of Polyethylene and Polytetrafluoroethylene on Their Tribological Response. Tribology Letters 42, 193201 (2011).

14. Barry, P. R., Chiu, P. Y. et al., Effect of Temperature on the Friction and Wear of PTFE by Atomic-Level Simulation. Tribology Letters 58, 50 (2015).

15. Li, C. \& Strachan, A., Molecular dynamics predictions of thermal and mechanical properties of thermoset polymer EPON862/DETDA. Polymer 52, 2920-2928 (2011).

16. Fang, Q., Tian, Y. et al., Revealing the deformation mechanism of amorphous polyethylene subjected to cycle loading via molecular dynamics simulations. RSC Adv. 8 (56), 32377-32386 (2018).

17. Li, C., Browning, A. R. et al., Atomistic simulations on multilayer graphene reinforced epoxy composites. Composites Part A: Applied Science and Manufacturing 43, 1293 1300 (2012).

18. Li, Y., Wang, Q. \& Wang, S., A review on enhancement of mechanical and tribological properties of polymer composites reinforced by carbon nanotubes and graphene sheet: Molecular dynamics simulations. Composites Part B: Engineering 160, 348-361 (2019).

19. Moghadam, A. D., Omrani, E. et al., Mechanical and tribological properties of selflubricating metal matrix nanocomposites reinforced by carbon nanotubes (CNTs) and graphene - A review. Composites Part B: Engineering 77, 402-420 (2015).

20. Zhan, S., Xu, H. et al., Molecular dynamics simulation of microscopic friction mechanisms of amorphous polyethylene. Soft Matter 15 (43), 8827-8839 (2019).

21. King, M. A., Blundell, D. J. et al., Modelling Studies of Crystalline PEEK. Molecular Simulation 4, 3-13 (1989).

22. Chen, C. L., Lee, C. L. et al., Molecular Dynamics Simulation of a Phenylene Polymer. 3. PEEK. Macromolecules 27, 7872-7876 (1994).

23. Pisani, W. A., Radue, M. S. et al., Multiscale modeling of PEEK using reactive molecular dynamics modeling and micromechanics. Polymer 163, 96-105 (2019). 
24. Cruz-Chú, E. R., Villegas-Rodríguez, G. J. et al., Mechanical characterization and induced crystallization in nanocomposites of thermoplastics and carbon nanotubes. npj Computational Materials 6, 151 (2020).

25. Jorgensen, W. L., Maxwell, D. S. \& Tirado-Rives, J., Development and Testing of the OPLS All-Atom Force Field on Conformational Energetics and Properties of Organic Liquids. Journal of the American Chemical Society 118, 11225-11236 (1996).

26. Price, M. L. P., Ostrovsky, D. \& Jorgensen, W. L., Gas-phase and liquid-state properties of esters, nitriles, and nitro compounds with the OPLS-AA force field. Journal of Computational Chemistry 22, 1340-1352 (2001).

27. Jorgensen, W. L., Chandrasekhar, J. et al., Comparison of simple potential functions for simulating liquid water. The Journal of Chemical Physics 79, 926-935 (1983).

28. Plimpton, S., Fast Parallel Algorithms for Short-Range Molecular Dynamics. Journal of Computational Physics 117, 1-19 (1995).

29. Stukowski, A., Visualization and analysis of atomistic simulation data with OVITO-the Open Visualization Tool. Modelling and Simulation in Material Science and Engineering 18 (2010).

30. Mehmet-Alkan, A. A. \& Hay, J. N., The crystallinity of poly(ether ether ketone). POLYMER 33 (16), 3527-3530 (1992).

31. Geier, S., Schmitz, H. et al., in Kunststoffe: Eigenschaften und Anwendungen, edited by Elsner, P., Eyerer, P. \& Hirth, T. (Springer Berlin Heidelberg, Berlin, 2012), pp. 1151201.

32. Shinoda, W., Shiga, M. \& Mikami, M., Rapid estimation of elastic constants by molecular dynamics simulation under constant stress. Phys. Rev. B 69 (13), 134103 (2004).

33. Lovinger, A. J. \& Davis, D. D., Electron- microscopic investigation of the morphology of a melt- crystallized polyaryletherketone. Journal of Applied Physics 58, 2843-2853 (1985).

34. Pastewka, L., Jacobs, T. \& Robbins, M. O., contact.engineering, Available at https://contact.engineering/.

35. Stanley, H. M. \& Kato, T., An FFT-Based Method for Rough Surface Contact. Journal of Tribology 119, 481-485 (1997).

36. Pastewka, L., Sharp, T. A. \& Robbins, M. O., Seamless elastic boundaries for atomistic calculations. Phys. Rev. B 86 (7), 075459 (2012).

37. Polonsky, I. A. \& Keer, L. M., A numerical method for solving rough contact problems based on the multi-level multi-summation and conjugate gradient techniques. Wear 231, 206-219 (1999).

38. Richards, F. M., Areas, volumes, packing and protein structure. Annual Review of Biophysics and Bioengineering 6, 151-176 (1977).

39. Ma, C.-C. M. \& Yur, S.-W., Environmental effects on the water absorption and mechanical properties of carbon fiber reinforced PPS and PEEK composites. Part II. Polymer Engineering \& Science 31, 34-39 (1991).

40. Tiwari, A., Wang, A. et al., Contact Mechanics for Solids with Randomly Rough Surfaces and Plasticity. Lubricants 7 (90) (2019). 


\section{Figures}
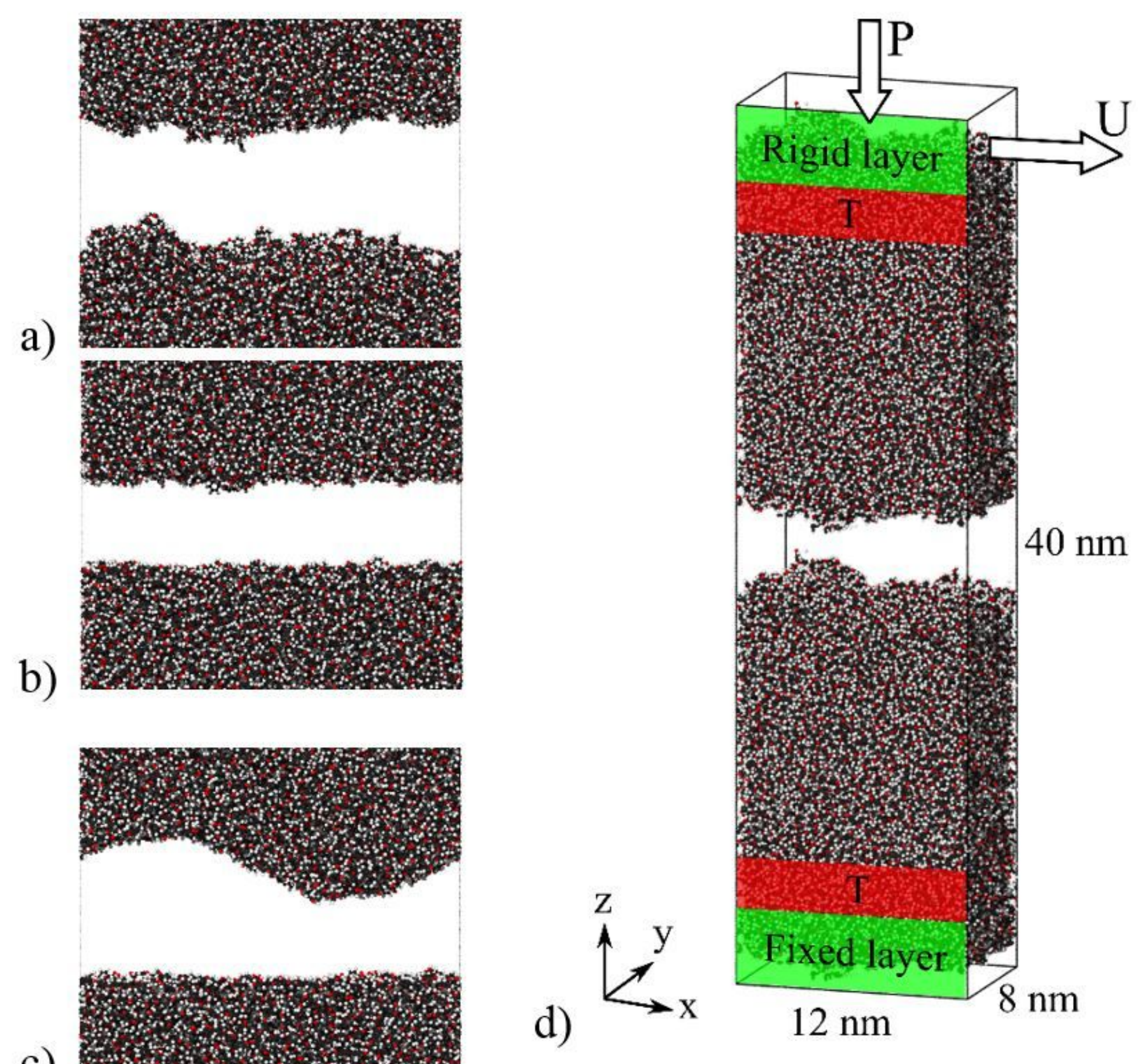

c) W

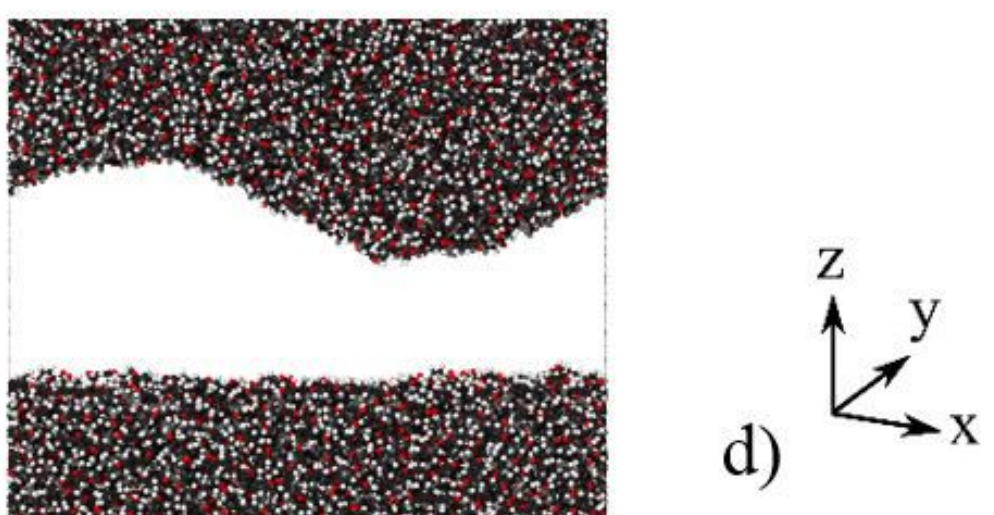

Figure 1

a-c) Amorphous PEEK surfaces: nanorough with hRMS $=4.3 \AA$, smooth with hRMS $=2.3 \AA$, and a sinusoidal geometry with $2 \mathrm{~nm}$ amplitude and $12 \mathrm{~nm}$ wavelength. d) Molecular dynamics sliding system. 
a)

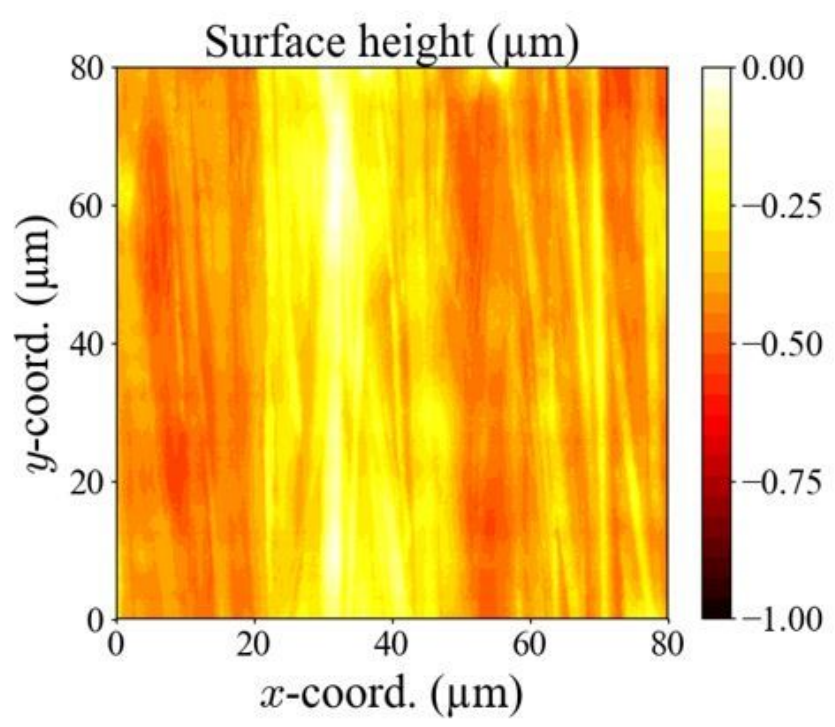

c)

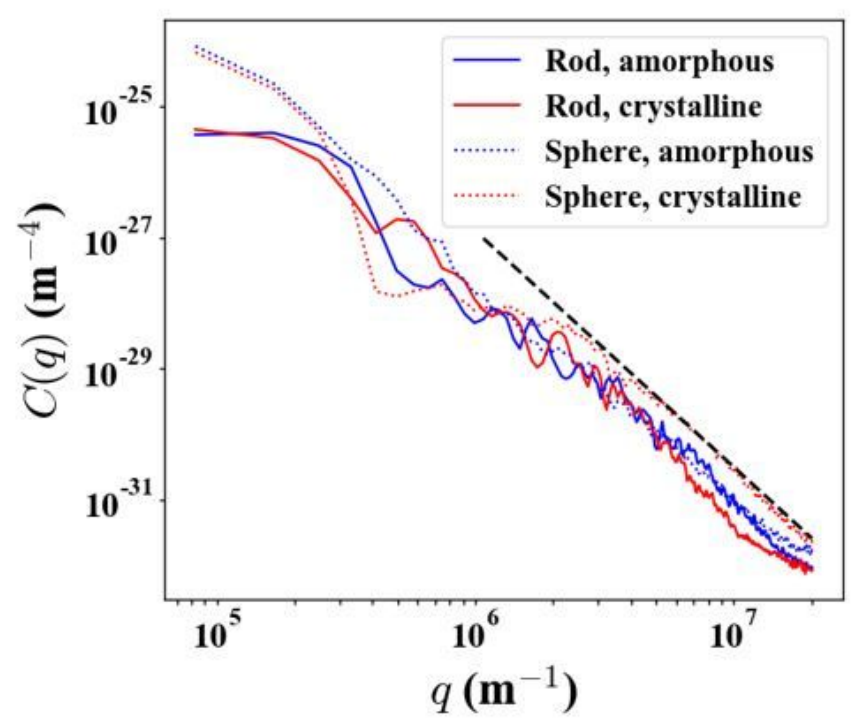

b)

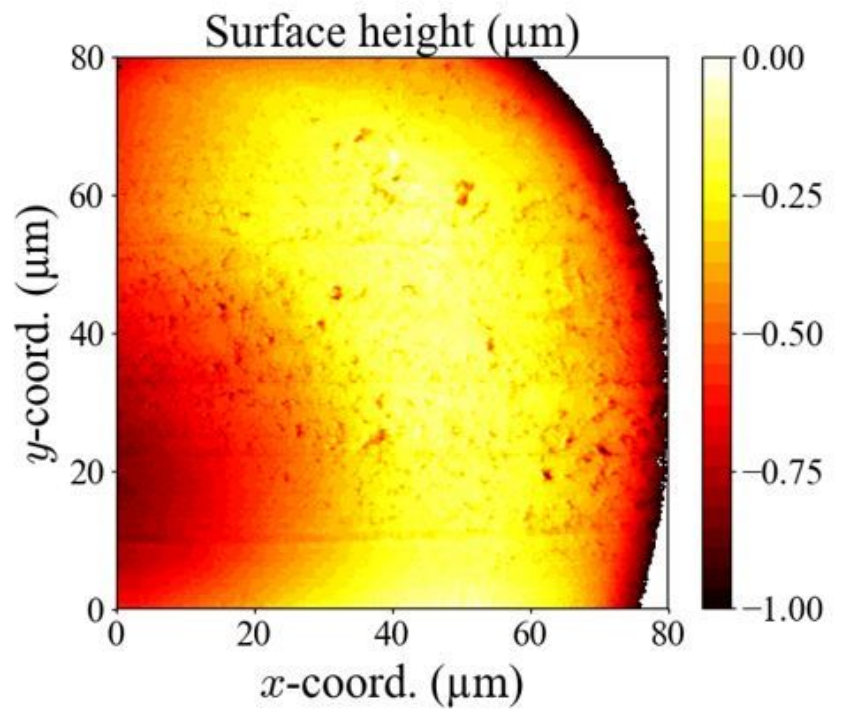

Figure 2: AFM scans of surface roughness of a) a traction rod, used as plane, and b) a sphere with $1.5 \mathrm{~mm}$ radius used in the tribometer experiments. The AFM resolution is $0.16 \mu \mathrm{m}$ in both directions. c) Radially averaged power spectral densities of the surface topographies. The dashed line indicates a Hurst exponent of $H=0.8$.

Figure 2

AFM scans of surface roughness of a) a traction rod, used as plane, and b) a sphere with $1.5 \mathrm{~mm}$ radius used in the tribometer experiments. The AFM resolution is $0.16 \mu \mathrm{m}$ in both directions. c) Radially averaged power spectral densities of the surface topographies. The dashed line indicates a Hurst exponent of $\mathrm{H}=0.8$. 
a)

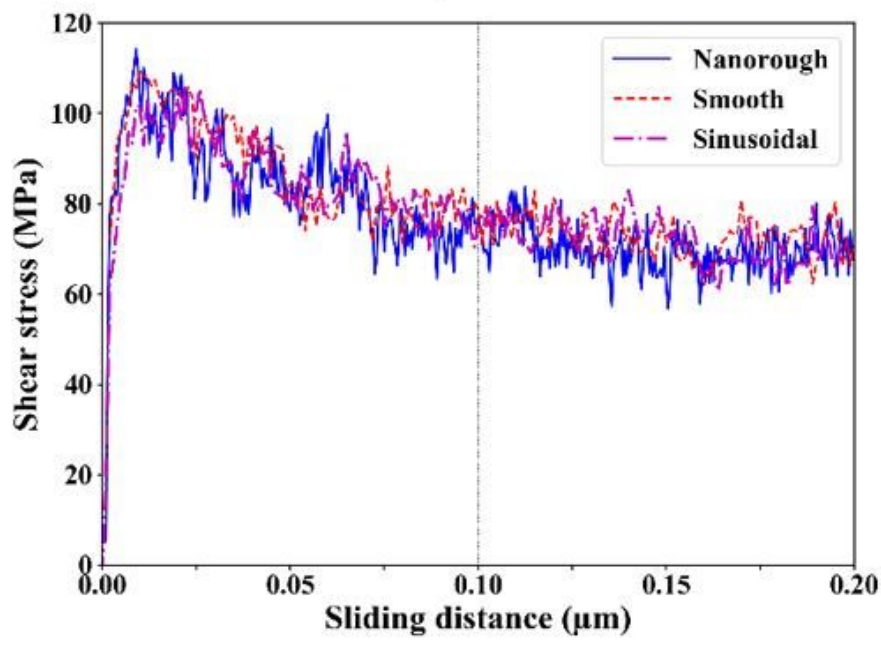

c)

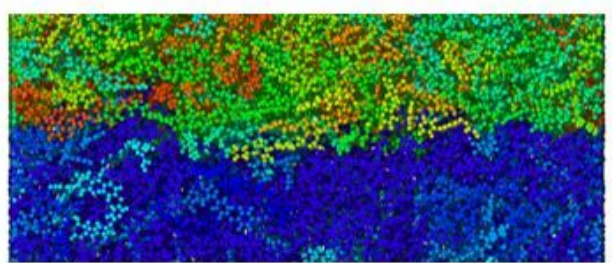

b)

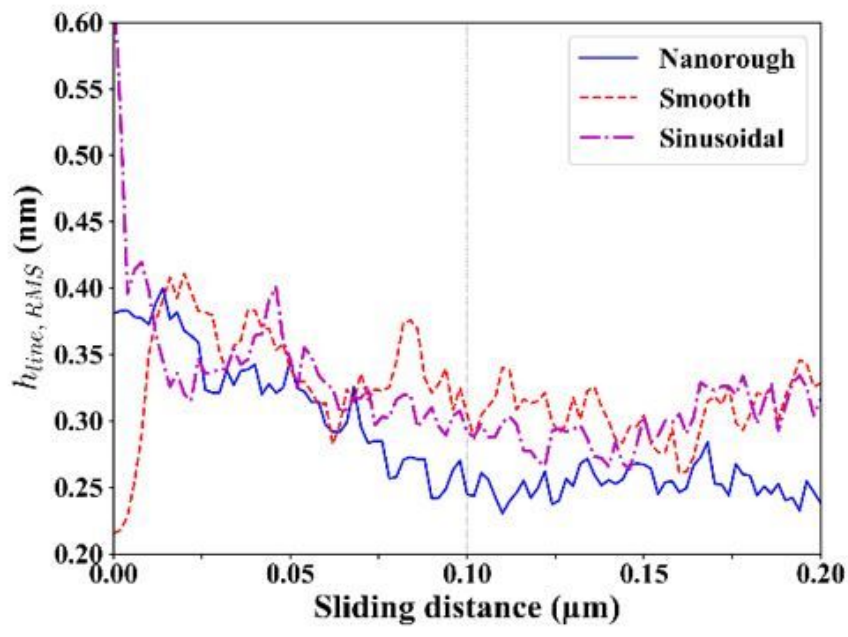

d)

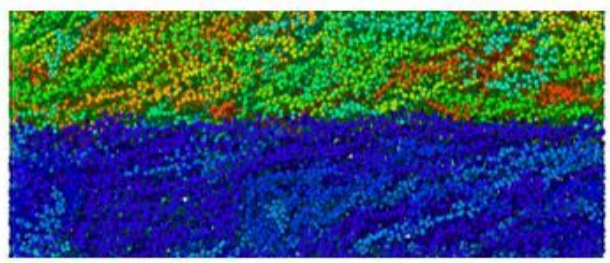

Figure 3

a) Shear stress and b) line RMS roughness for three amorphous PEEK-PEEK interfaces as a function of the sliding distance $(P=100 \mathrm{MPa}, \mathrm{U}=100 \mathrm{~m} / \mathrm{s}, \mathrm{T}=300 \mathrm{~K})$. After $0.1 \mu \mathrm{m}$ sliding, steady state friction is reached. c) Snapshot of the nanorough interface at the beginning of shearing and d) after $0.1 \mu \mathrm{m}$ sliding distance. Different colors indicate different oligomer chains (top surface red to green, bottom surface cyan to blue). 
a)

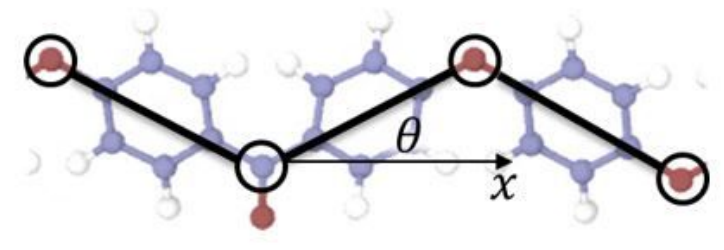

b)

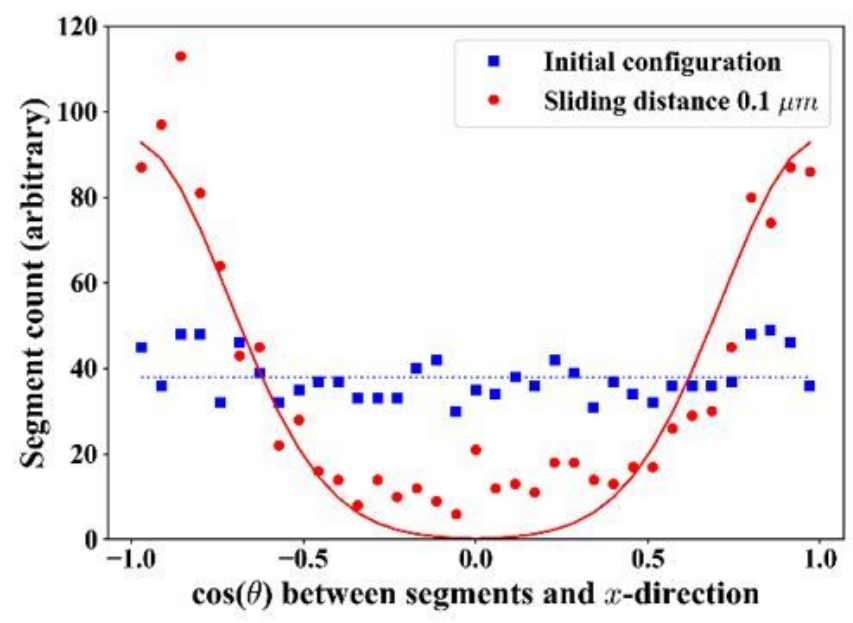

c)

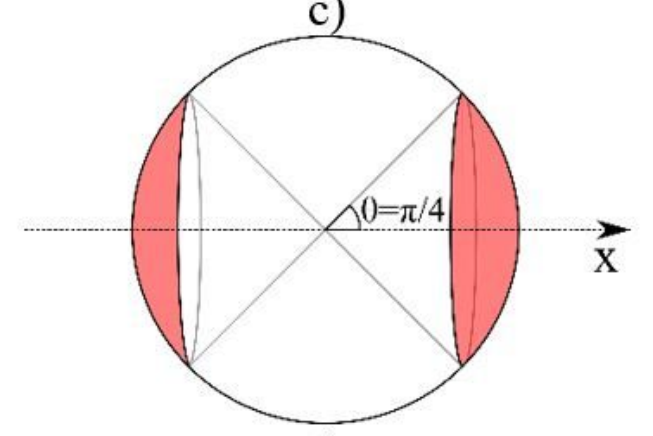

d)

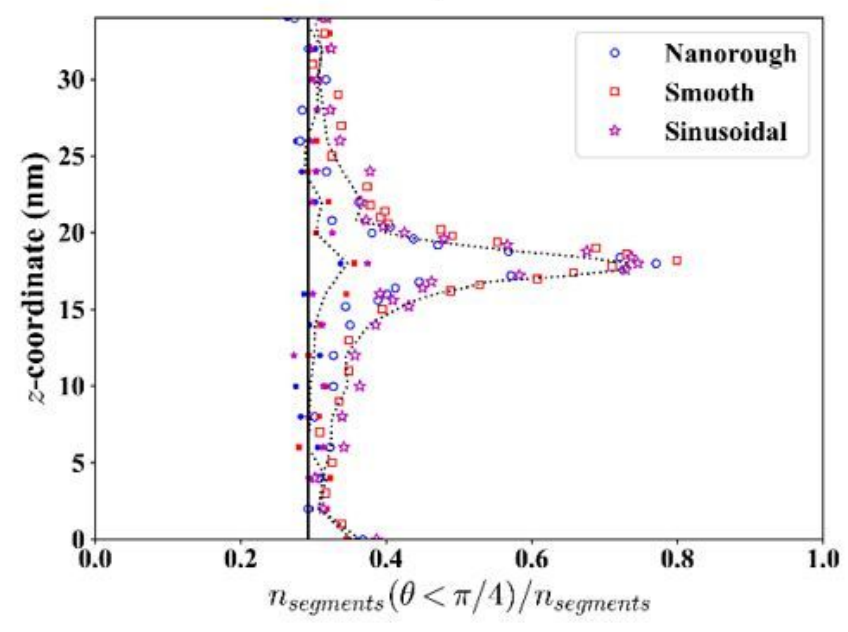

Figure 4

a) Representation of simplified PEEK chain used in the analysis. b) Segment angle distribution at the sliding interface. The continuous red line is a Gaussian curve with standard deviation corresponding to | $\theta \mid=\pi / 4$. c) Aligned segments are contained within two spherical sectors featuring an aperture angle of $\pi / 4$ with respect to the $x$-direction. In systems without preferential orientation, the percentage of the aligned is given by the surface of the two spherical sectors, divided by the sphere surface. This gives 1$\cos (\pi / 4) \approx 0.29$. d) Number of aligned segments over the system height, with the sliding interface being located at $z=18 \mathrm{~nm}$. Full markers correspond to the beginning of shearing, whereas empty markers indicate steady state shear results. 


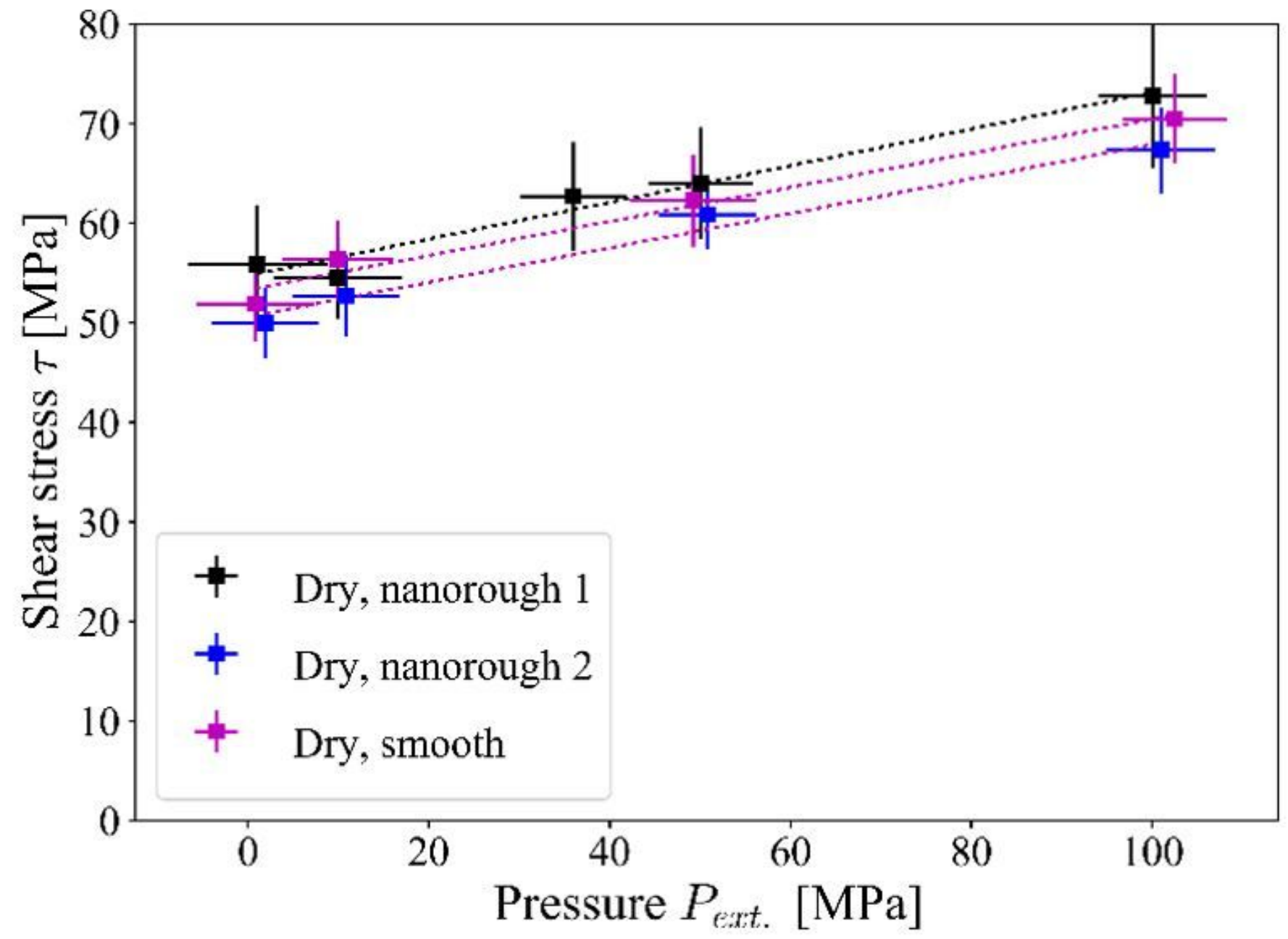

Figure 5

Shear stress in dry amorphous PEEK-PEEK interfaces as a function of the applied pressure $(U=100 \mathrm{~m} / \mathrm{s}$, $\mathrm{T}=300 \mathrm{~K})$. The dotted lines represent linear fits of the data points. Nanorough 2 represents an additional realization of a nanorough dry interface, obtained by removing water from one of the systems in Section 3.2. 
a)

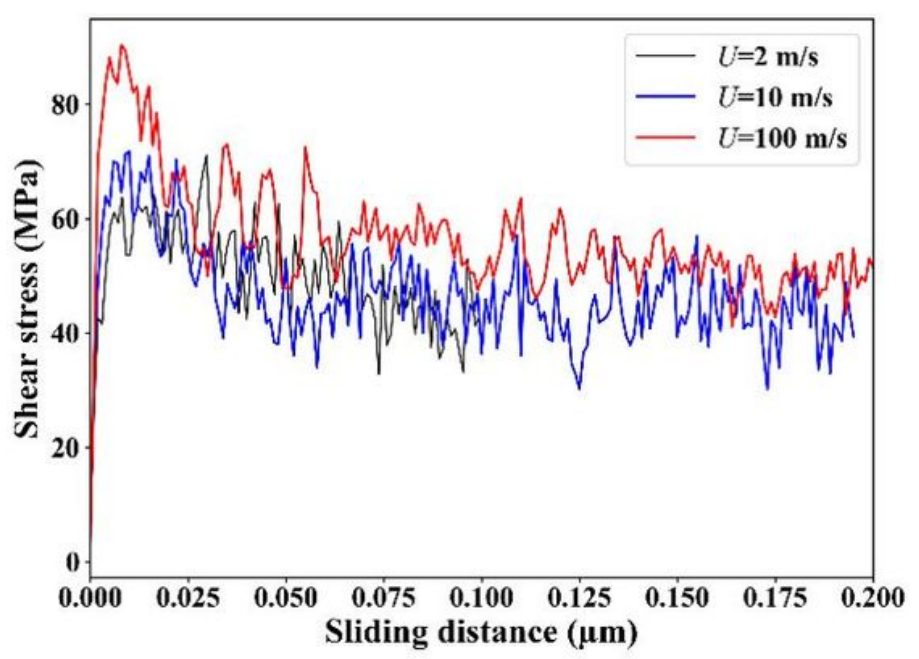

b)

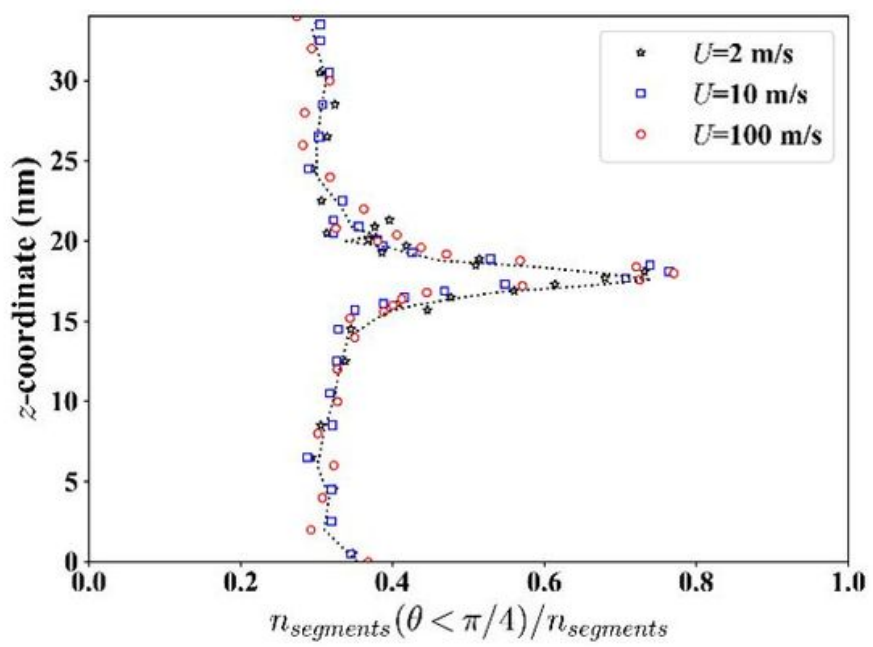

Figure 6

a) Shear stress as a function of the sliding distance at different sliding velocities and low load ( $P=1 \mathrm{MPa}$, $\mathrm{T}=300 \mathrm{~K})$. b) Chain alignment after a sliding distance of $0.1 \mu \mathrm{m}$ for three shear velocities.

a)

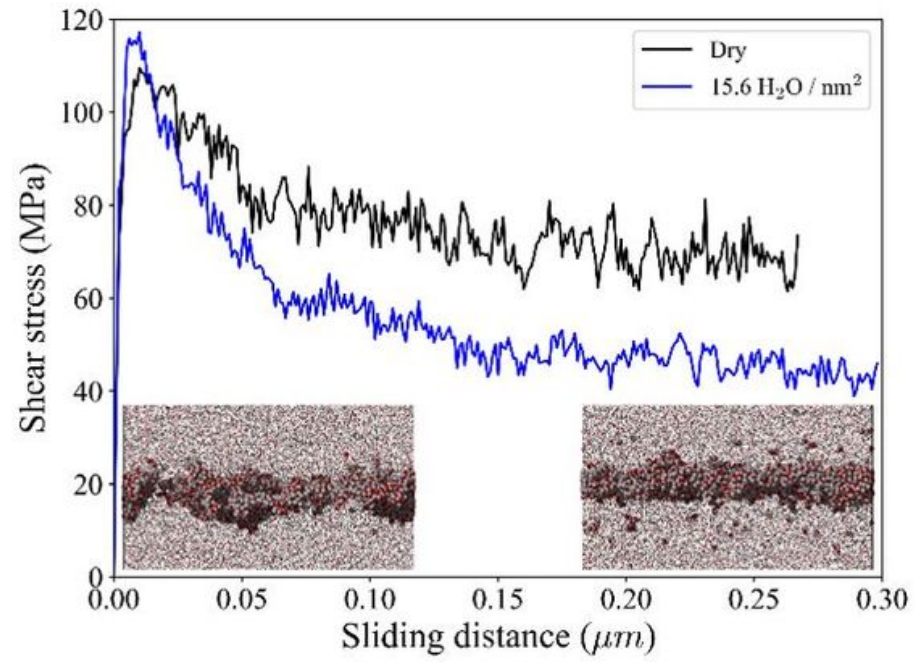

b)

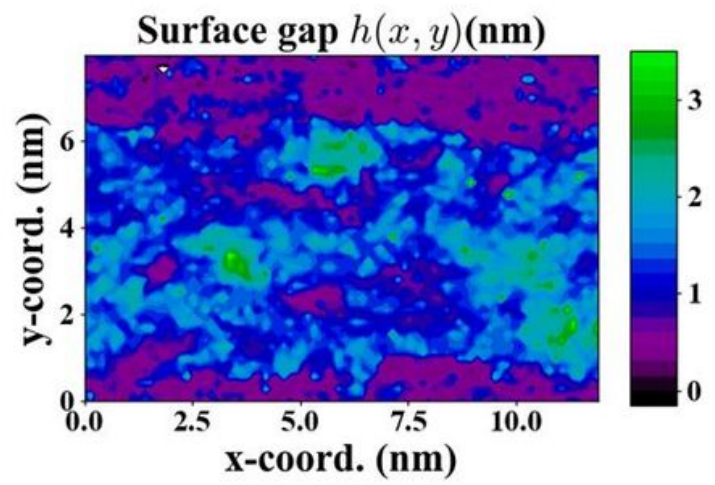

\section{Figure 7}

Shear stress as function of the sliding distance for a water-lubricated interface $(P=100 \mathrm{MPa}, \mathrm{U}=100 \mathrm{~m} / \mathrm{s}$, $\mathrm{T}=300 \mathrm{~K}, 15.6 \mathrm{nH} 2 \mathrm{O} / \mathrm{nm}^{2}$ ). The curve for a dry case under the same conditions is also shown for comparison. The insets depict the repartition of the water molecules at the sliding interface before sliding and after steady state is reached. b) Intersurface gap in steady state shearing. Purple domains correspond to PEEK-PEEK contact, whereas blue and green areas indicate a water nanochannel aligned in the shearing direction. 
a)

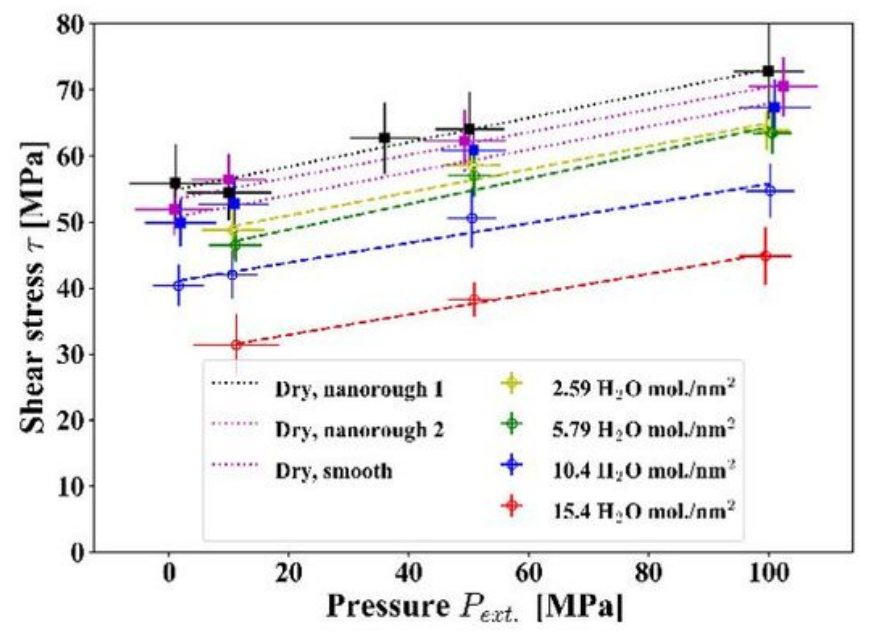

b)

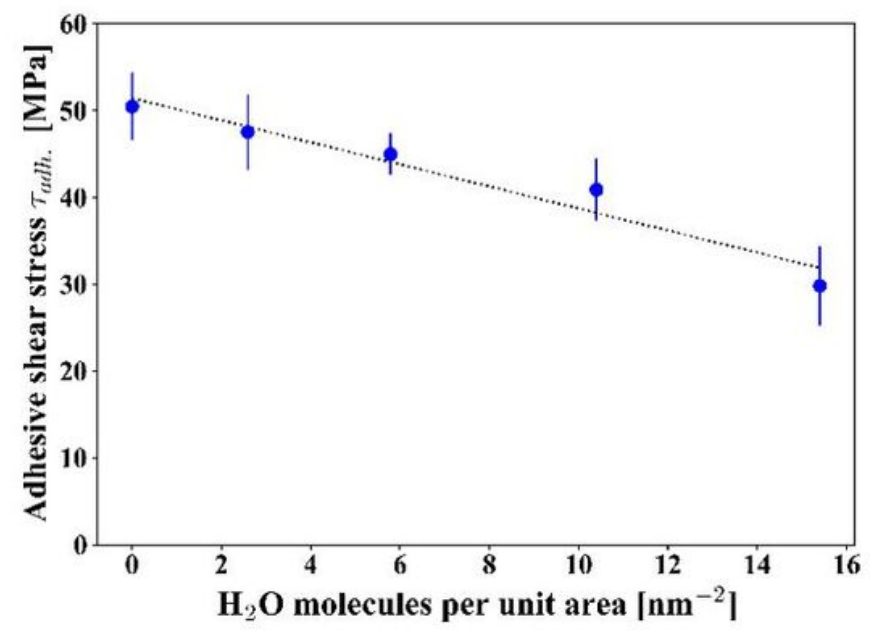

\section{Figure 8}

a) Shear stress in dry and water-lubricated PEEK-PEEK interfaces as a function of the applied pressure $(\mathrm{U}=100 \mathrm{~m} / \mathrm{s}, \mathrm{T}=300 \mathrm{~K})$. b) Dependence of the adhesive stress $\mathrm{\tau}$ \adh. on the amount of water confined between PEEK surfaces. The dotted lines represent linear fits to the data points. 


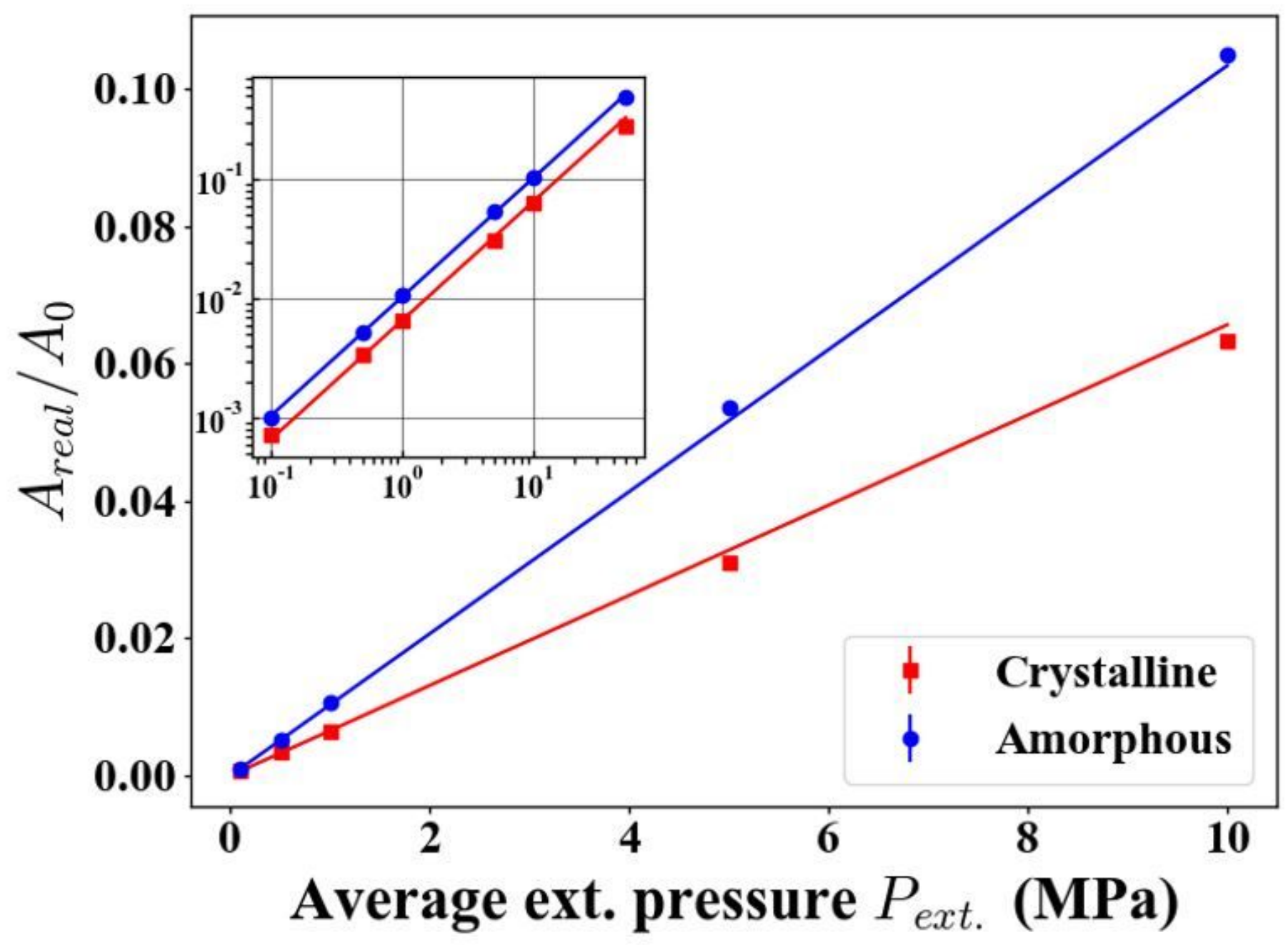

Figure 9

Relative contact area as a function of the external pressure. The inset features the same data with logarithmic axes. Both straight lines in the inset have a slope of 1 , indicating linearity between relative contact area and applied load over several orders of magnitude. The external pressure up to $50 \mathrm{MPa}$ (inset) over the considered surface area corresponds to a normal load up to $0.32 \mathrm{~N}$. 
a) Amorphous PEEK pairing

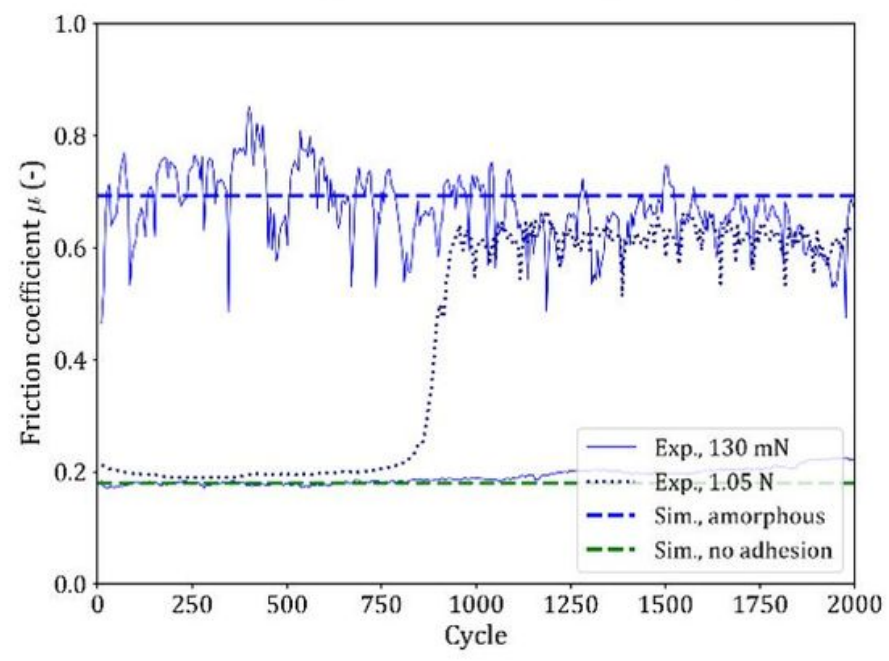

c)

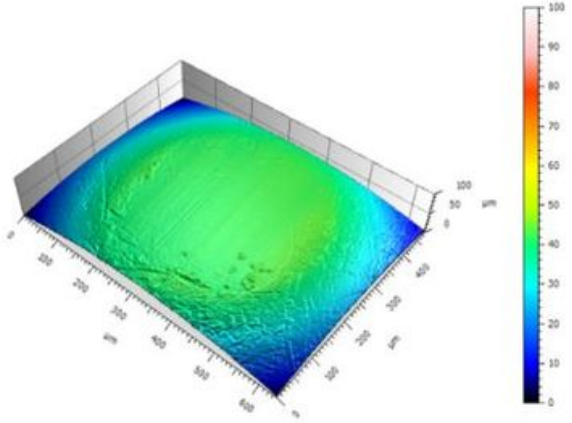

b) Crystalline PEEK pairing
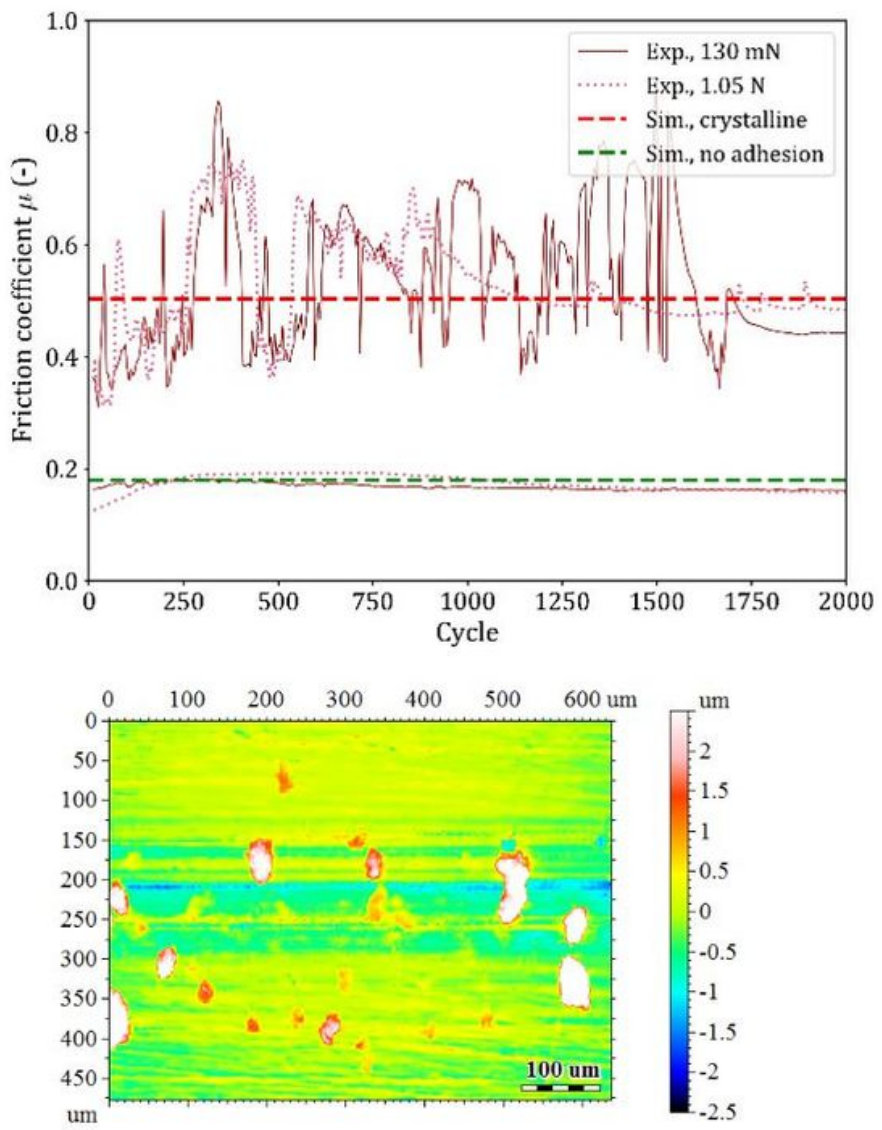

Figure 10

a-b) Experimental friction coefficient as a function of the number of reciprocating cycles for the amorphous and crystalline pairings, respectively. c) Confocal microscope image of a worn crystalline sphere and material transfer to the rod after a high friction run at $0.310 \mathrm{~N}$ load. 